\title{
Tissue-specific impacts of aging and genetics on gene expression patterns in humans
}

\author{
Ryo Yamamoto ${ }^{1,2, A}$, Ryan Chung ${ }^{3, A}$, Juan Manuel Vazquez ${ }^{1}$, Huanjie Sheng ${ }^{1}$, Philippa Steinberg ${ }^{1}$, Nilah M loannidis ${ }^{3,4}$, and \\ Peter H Sudmant ${ }^{1,3}$ \\ ${ }^{1}$ Department of Integrative Biology, University of California, Berkeley \\ ${ }^{2}$ Bioinformatics Interdepartmental Program, University of California, Los Angeles \\ ${ }^{3}$ Center for Computational Biology, University of California, Berkeley \\ ${ }^{4}$ Department of Electrical Engineering and Computer Sciences, University of California, Berkeley
}

Age is the primary risk factor for many common human dis- 45 eases including heart disease, Alzheimer's dementias, cancers, 46 and diabetes. Determining how and why tissues age differently is key to understanding the onset and progression of such pathologies. Here, we set out to quantify the relative contributions of genetics and aging to gene expression patterns from data collected across 27 tissues from 948 humans. We show that ${ }^{50}$ gene expression patterns become more erratic with age in sev- 51 eral different tissues reducing the predictive power of expres- 52 sion quantitative trait loci. Jointly modelling the contributions 53 of age and genetics to transcript level variation we find that the 54 heritability $\left(h^{2}\right)$ of gene expression is largely consistent among tissues. In contrast, the average contribution of aging to gene expression variance varied by more than 20 -fold among tissues with $R_{\text {age }}^{2}>h^{2}$ in 5 tissues. We find that the coordinated decline of mitochondrial and translation factors is a widespread signature of aging across tissues. Finally, we show that while in general the force of purifying selection is stronger on genes ${ }^{60}$ expressed early in life compared to late in life as predicted by Medawar's hypothesis, a handful of highly proliferative tissues 6 exhibit the opposite pattern. In contrast, gene expression varia- 63 tion that is under genetic control is strongly enriched for genes 64 under relaxed constraint. Together we present a novel frame- 65 work for predicting gene expression phenotypes from genetics and age and provide insights into the tissue-specific relative contributions of genes and the environment to phenotypes of aging.

A RY and RC contributed equally to this work

aging | genetics| eQTL | Medawar

Correspondence: psudmant@berkeley.edu,nilah@berkeley.edu

\section{${ }_{31}$ Introduction}

biological processes across tissues and identifying how genetic variation influences such changes.

At the organismal level, age-associated changes in the heterogeneity of gene expression between individuals have been observed for a handful of genes in humans (3). In an analysis of gene expression in monozygotic (identical) twins, 42 genes showed age-associated differences in gene expression, suggesting a role for the environment in modulating gene expression with age $(2,3)$. Similarly, the proportion of expression quantitative trait loci (eQTLs) detected from blood in 70 year olds declined by $2.7 \%$ when they were resampled at 80 years old (4). However, the extent of this phenomenon, both across genes and tissues, remains unclear (5). Age-associated increases in the heterogeneity of gene expression have also been observed at the level of individual cell-to-cell variation; however, only some cell types appear to be impacted (6). In a recent study of immune T-cells from young and aged individuals, no difference in cell-to-cell variability was observed in unstimulated cells, however, upon immune activation the older cells appeared more heterogeneous (7). It is not known why some cell-types and not others may be more likely to exhibit increased cellular variability.

The relationship between the age at which a specific gene is expressed and the force of purifying selection has also recently been explored across a number of species $(8,9)$. These analyses have broadly confirmed that, on average, genes expressed later in life are under less constraint compared to those expressed early in life. However, how these patterns vary across different tissues and are impacted by genetic variation has not been systematically explored.

Here we set out to understand how aging affects the molecular heterogeneity of gene expression and to model the relative impact of age and genetic variation on this phenotype across tissues. First, using gene expression data from 948 individuals in GTEx V8 (10), we show that eQTLs are less predictive in older individuals, however to a different extent across various tissues. We show that gene expression heterogeneity between individuals increases with age in these tissues. Using a regularized linear model-based approach to jointly model the impact of both age and genetic variation on gene expression we find that while the average heritability of gene expression is consistent across tissues, the average contribution of age varies substantially. Furthermore, while the genetic regulation of gene expression is similar across tissues, 
age-associated changes in gene expression are highly tissue- ${ }_{144}$ specific in their action. We use this joint model to identify 145 each gene's age of expression and show that while in most ${ }_{146}$ tissues late-expressed genes do tend to be under more relaxed ${ }_{147}$ selective constraint, among a handful of highly proliferative ${ }_{148}$ tissues the opposite trend holds.

\section{Results}

Expression quantitative trait loci exhibit varying predictive power in old and young individuals across several different tissues. To gain insight into how gene regulatory programs might be impacted by aging we analyzed transcriptomic data collected across multiple tissues from $948_{157}$ humans (GTEx version 8) (10). We hypothesized that aging might dampen the effect of expression quantitative trait loci (eQTLs) due to factors such as increased environmental variance or molecular infidelity (Fig. 1A). To test this hypothesis ${ }_{16}$ we first classified individuals into young and old age groups conservatively grouping individuals above and below the median age (55 years old, Fig. S1), respectively, restricting our ${ }_{164}$ analyses to tissues with at least 100 individuals in both groups (27 tissues in total, Fig. S2, Table S1). In each tissue we down-sampled to match the sample size of old and young individuals while additionally controlling for co-factors such ${ }_{168}$ as ancestry and technical confounders (methods). Of note, a common approach to controlling for unobserved confounders in large gene expression experiments is to probabilistically infer hidden factors using statistical tools such as PEER (11). We noticed that many of the GTEx PEER factors were significantly correlated with sample age, with the top three correlated PEER factors having a Pearson $r$ of $0.33,-0.21$, and -0.15 (Fig. S3). To prevent loss of age related variation, we recalculated a corrected set of PEER factors that were independent of sample age (Methods). We then assessed the significance of GTEx eQTLs in the young and old cohorts respectively, comparing the distribution of $\mathrm{P}$-values over all genes between old and young individuals (Fig. 1A). In 20 out of $27(74 \%)$ of the assessed tissues, the P-value distribution was significantly different between young and old individuals with genotypes more predictive of expression in younger individuals in 12/20 cases (e.g. Fig. 1D). These results were largely identical when the analyses were performed with the original non-corrected PEER factors (18/27 tissues, Fig. S4). ${ }^{185}$ These results suggest that the predictive power of eQTLs is ${ }^{186}$ impacted by the sample age across the vast majority of tis- ${ }^{187}$ sues. Furthermore this effect is more pronounced in older ${ }^{188}$ samples compared with younger samples.

Age-associated increases in gene expression hetero- ${ }^{19}$ geneity reduce gene expression heritability. We hypoth- ${ }^{192}$ esized that the reduced predictive power of eQTLs in some ${ }^{193}$ older tissues might be in part due to an overall increase in ${ }^{194}$ expression heterogeneity in these tissues, potentially as a re- 195 sult of increased environmental variance. To test if such an 196 effect would broadly affect expression across all genes in a 197 tissue (Fig. 2A) we calculated the distribution of pairwise ${ }_{198}$ distances among individual's tissue-specific gene expression 199 profiles using the Jensen-Shannon Divergence (JSD) $(12,13)$ as a distance metric. The JSD is a robust distance which is less impacted by outliers compared to other methods (e.g. Euclidean distance) (13). Comparing the distribution of pairwise differences in transcriptional profiles within distinct age groups allows us to determine if gene expression signatures are more similar among younger individuals versus among older individuals.

We compared the mean difference in gene expression distances among old and young individuals as well as the slope of the inter-individual JSD and when grouping individuals into six bins spanning 20-80 years old (see methods, Fig. 2B, $2 \mathrm{C}$ ). These two strategies yielded highly similar results (Fig. $2 \mathrm{~B} R=0.8$ ) and identified a cluster of 12 tissues exhibiting robust increases in the average inter-individual expression distance as a function of age (e.g. Fig. 2C). Our JSD analysis of old and young individuals was also negatively correlated with the results from our analysis of eQTLs across old and young individuals (Fig. S5, $\mathrm{R}=-0.48, \mathrm{P}=0.01$ ) highlighting that tissues with age-associated increases in inter-individual heterogeneity were likely to also exhibit reductions in the proportion of variance described by eQTLs. Conversely, tissues in which eQTLs explained a higher proportion of gene expression variance in older individuals exhibited a decrease in inter-individual gene expression variation.

To expand our eQTL analyses to account for the combined impact of nearby SNPs, we utilized the multi-SNP regularized linear model of PrediXcan (14). This model has the benefit of combining genetic effects across many loci, instead of examining just a single eQTL variant. This combined genetic contribution to gene expression variance results in an estimate of the heritability $\left(h^{2}\right)$ for each gene. We applied this model independently in old and young individuals to quantify $h^{2}$ and found that the average per-gene difference in $h^{2}$ between old and young individuals was strongly negatively correlated with the difference in JSD between samples ( $\mathrm{R}=0.6, \mathrm{P}=9.9 \mathrm{e}-4$, Fig. 2D, Fig. S6). Together these results suggest that across numerous tissues aging is associated with an overall increase in gene expression heterogeneity. This increased expression variance drives a reduction in the average heritability of gene expression across these tissues.

Jointly modeling the impact of age and genetics on gene expression identifies distinct, tissue-specific patterns of aging. A more powerful approach to understand how both genetics and age impact gene expression variation is to jointly model these factors simultaneously. We set out to extend the regularized linear model to incorporate an age factor (Fig. 3A) allowing us to parse apart the individual contributions of genetics $\left(R_{\text {genetics }}^{2}\right.$ or $\left.h^{2}\right)$, age $\left(R_{\text {age }}^{2}\right)$, and the environment $\left(R_{\text {environment }}^{2}\right)$, to the expression variance of each gene (e.g. Fig. 3B, Fig. 3C). We define $R_{\text {environment }}^{2}$ as all sources of variation not captured by $h^{2}$ and $R_{\text {age }}^{2}$. Estimates of $h^{2}$ in our extended model were highly consistent with those in the original PrediXcan approach (Fig. S 7).

Employing our model across each tissue independently we find that average heritability of gene expression was largely 
A)
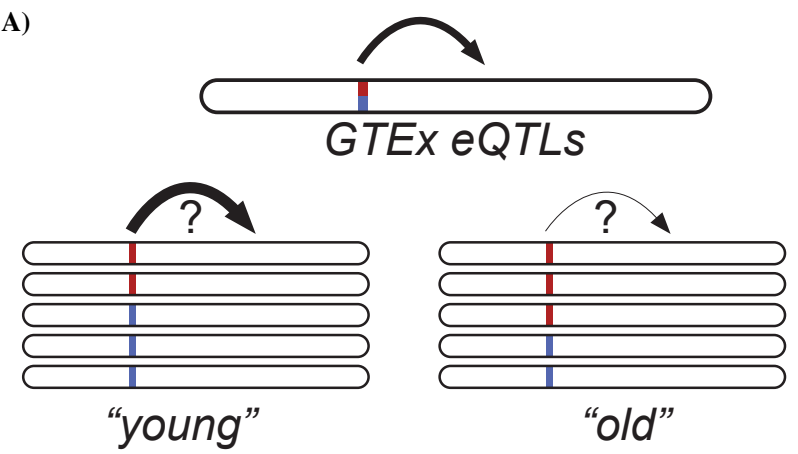

B)

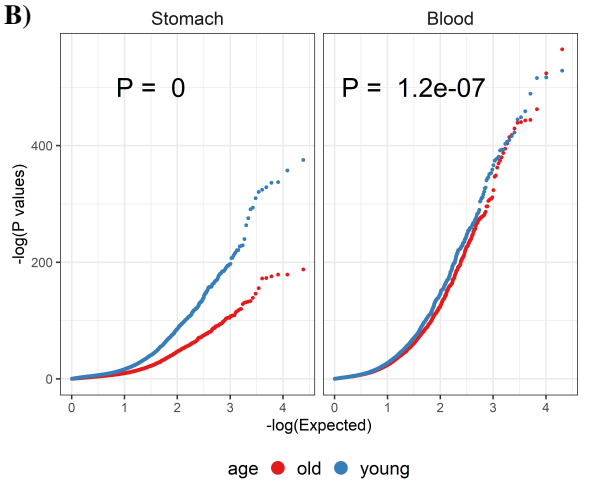

C)
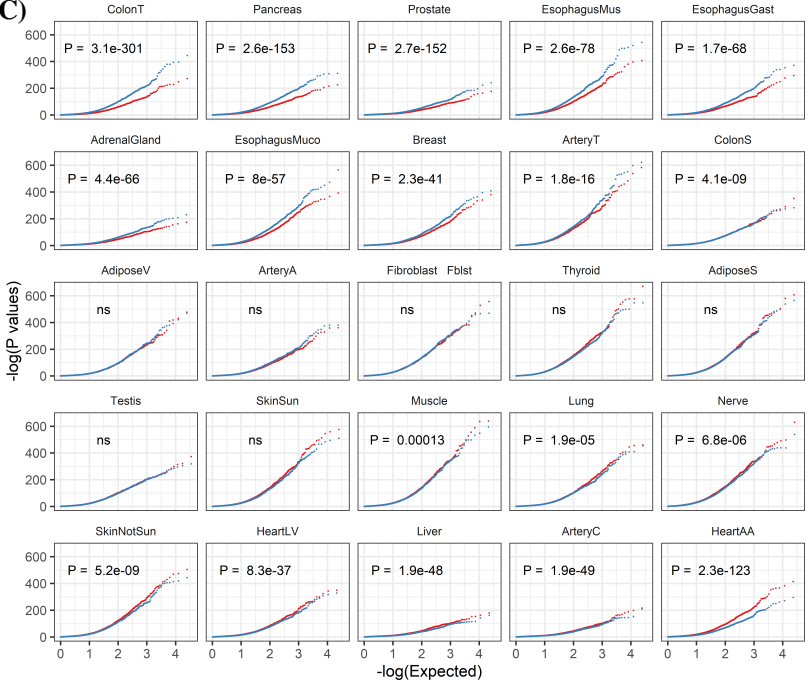
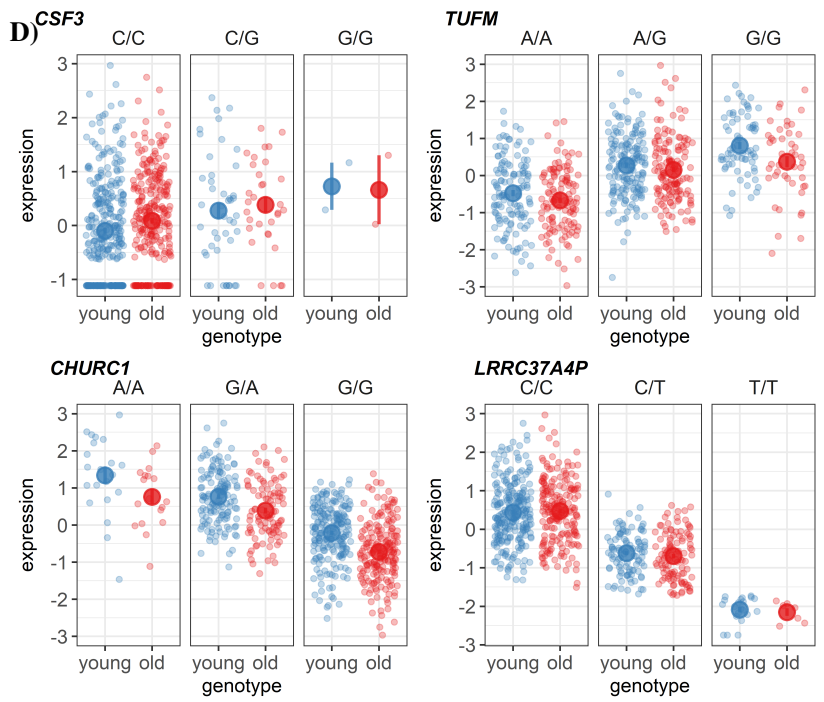

Fig. 1. eQTLs become less explanatory with age in many tissues. A) A hypothetical model of the differing power to detect eQTLs in old and young cohorts. B) $Q Q$ plots of eQTL p-values (plotted as - $\log (\mathrm{P})$ ) for old (red) and young (blue) individuals from a linear model correlating expression with the lead SNP for each gene in blood and stomach tissue and C) all other tissues (Table S2). D) Examples of gene expression binned by genotype and age for four genes in which eQTLs are less explanatory in older individuals in whole blood.

consistent among tissues ranging from 2.9\%-5.7\% with $40 \% 222$ of genes having an $h^{2}>10 \%$ in at least one tissue (Fig. 3D, 223 S8). Thus, while the variation in expression of many indi- 224 vidual genes is strongly influenced by genetics, on average, 225 genetics explains a small proportion of overall gene expres- 226 sion variation. In contrast, the average contribution of aging ${ }_{227}$ to gene expression varied more than 20-fold among tissues from $0.4 \%-7.9 \%$ with the average $R_{\text {age }}^{2}$ greater than the av- 228 erage $h^{2}$ in 5 tissues. Among these 5 tissues the expression 229 of $39-54 \%$ of genes was more influenced by age than by ge- 230 netics (i.e. $R_{\text {age }}^{2}>h^{2}$, Fig. S9) and across all tissues $45 \%$ of 231 genes had an $R_{\text {age }}^{2}>10 \%$ in at least one tissue. Assessing the ${ }^{232}$ tissue-specificity of these trends on a per-gene basis we found ${ }^{233}$ while the estimated heritability of gene expression tended to ${ }^{234}$ be similar among different tissues, the age-associated compo- 235 nent exhibited significantly more tissue specificity $(\mathrm{P}<2.2 \mathrm{e}-236$ 16, Fig. 3E). We note that the widespread signatures of ${ }^{237}$ age-associated gene expression variance that we identify are ${ }^{238}$ virtually undetectable when using the GTEx-provided PEER ${ }^{239}$ factors. Just $1.84 \%$ of the age-associated genes we identify ${ }^{240}$ have nonzero age coefficient when using these GTEx PEER ${ }^{241}$ factors (Fig. S10). Our model thus widely expands the util- 242 ity of the GTEx dataset and exploration of critical biological signatures of aging. Together these results imply that ageassociated patterns of gene expression exhibit substantially more tissue specificity than those that are influenced by genetics and among several tissues age plays a much stronger role in driving gene expression patterns than genetics.

\section{Coordinated decline of mitochondrial and translation factors is a widespread signature of aging across tis-} sues. To understand the underlying biological implications of age-associated gene expression changes we applied gene set enrichment analysis (GSEA)(15) to each tissue independently, ranking genes either by the relative contribution of genetics $\left(h^{2}\right)$ or aging $\left(R_{\text {age }}^{2}\right)$. Comparing the distribution of P-values from enriched GO-annotations we found that pathways enriched for age-associated variance were substantially more enriched for significance than pathways associated with genetic-associated variance (e.g. Fig. 4A). We found more age-associated pathway enrichment even in tissues for which the average age-associated contribution to gene expression was low (e.g. Pancreas, Fig. S11). This implies that while age-associated changes in gene expression vary widely in their magnitude among tissues, these changes 

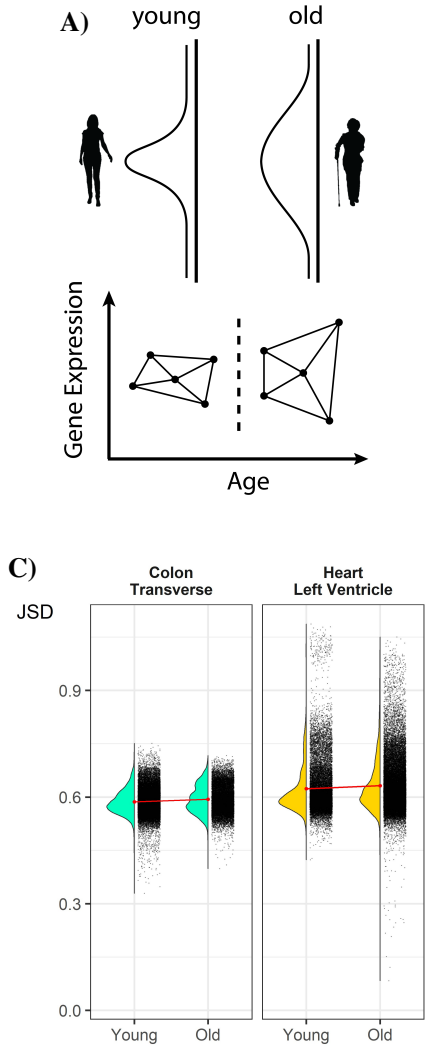
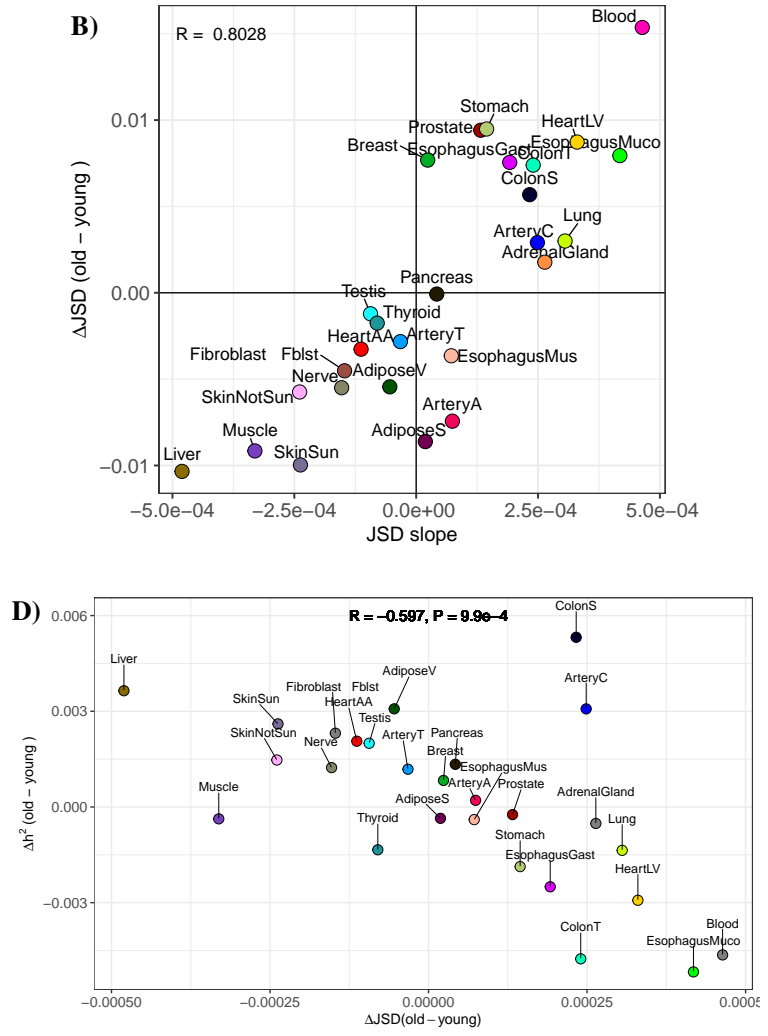

Fig. 2. Inter-individual gene expression heterogeneity increases with age for a subset of tissues. A) Hypothesized age-associated increase in gene expression heterogeneity (top) and our approach for quantifying the inter-individual expression distance with age using the Jensen Shannon Divergence metric (JSD) for age-binned individuals (bottom). B) Consistency of measuring the average age-associated change in gene expression heterogeneity across a tissue using a binary binning strategy (y-axis, $J S D_{\text {old }}-J S D_{\text {young }}$ ) or a 6 bin strategy (x-axis, slope of JSD across 6 bins). C) The distributions of JSD distances for two tissues in old and young bins. D) The relationship between gene expression heterogeneity and the difference in expression heritability between old and young individuals.

consistently impact critical biological processes. A GSEA 270 enrichment analysis of genes ranked by the tissue-averaged ${ }_{271}$ slope of the age-associated trend ( $\left.\beta_{\text {age }}\right)$ highlighted several 272 key aging-associated pathways. Pathways associated with 273 various mitochondrial and metabolic processes and transla- 274 tion were enriched for having $-\beta_{\text {age }}$ values, implying age- 275 associated decreases (Fig. 4B). A single immune pathway, 276 the interferon-gamma response was enriched having $+\beta_{\text {age }} 277$ values (Fig. 4B). An additional 18 immune pathways were 278 identified as having age-associated increases in gene expres- 279 sion using a more lenient significance threshold $(\text { FDR }<0.05)_{280}$ (Fig. S12). In contrast, no pathways were significantly enriched when genes were ranked by average $h^{2}$.

To further explore the functional impact of age-associated 282 gene expression changes we compared the $R_{\text {age }}^{2}$ of all ${ }_{283}$ nuclear-encoded mitochondrial genes $(\mathrm{n}=1120,(16))$, and 284 translation initiation, elongation, and termination factors, 285 across tissues (Fig. 4C, Fig. S13). Genes in these path- 286 ways were exceptionally enriched for age-associated gene ex- 287 pression across several tissues. In some cases $>10 \%$ of the 288 average expression variation of mitochondrial or translation 289 factor genes could be explained by age. $\beta_{\text {age }}$ was consis- 290 tently negative in these mitochondrial and translation factor 291 genes (Fig. 4D) highlighting that genes in these pathways 292 exhibit a systematic decrease in expression as a function of 293 age. Overall across tissues an average of $36 \%$ of all mi- 294 tochondrial genes (406/1120), and 35\% of translation factors $(119 / 337)$ exhibited age-associated declines, however in some tissues these proportions exceeded $60 \%$. In contrast, the only pathway associated with age-associated increases in expression, interferon-gamma response genes, was largely specific to blood and arterial tissue (Fig. 4C), likely due to the role of this pathway in immune cells. Together these results demonstrate that the coordinated decline of mitochondrial genes and translation factors is a widespread phenomenon of aging across several tissues with potential phenotypic consequences.

\section{Distinct evolutionary signatures of gene expression patterns influenced by aging and genetics. Evolution-} ary theory predicts that due to the increased impact of selection in younger individuals, genes that increase as a function of age $\left(\beta_{\text {age }}>0\right)$ should be under reduced selective constraint compared to genes that are highly expressed in young individuals $\left(\beta_{\text {age }}<0\right)$, a theory of aging known as Medawar's hypothesis (1) (Fig. 5A). Several recent studies have demonstrated the generality of this trend across species $(8,9,17)$ however the tissue-specificity of this theoretical prediction has not been explored. We sought to test the generality of this trend across different tissues by comparing $\beta_{\text {age }}$ with the level of constraint on genes, quantified as the probability loss of function intolerance ( $\mathrm{pLI}$ ) score from gnomAD 
A)

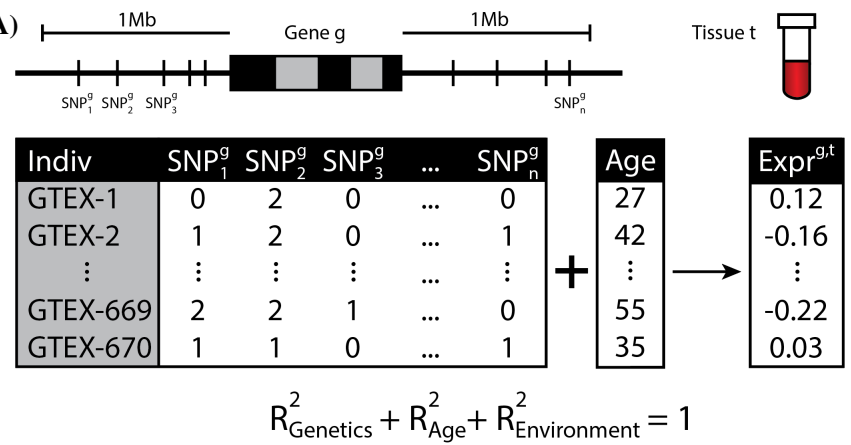

B)

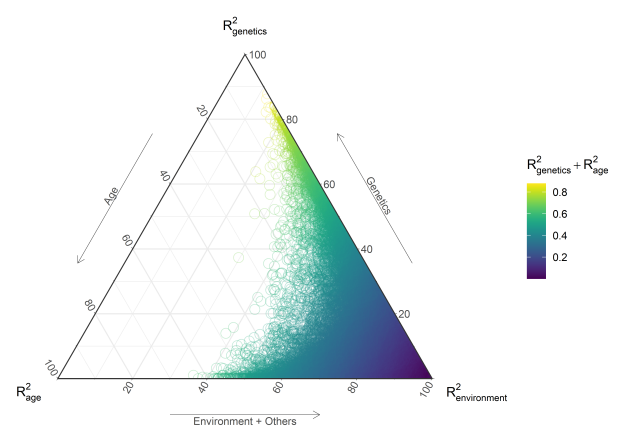

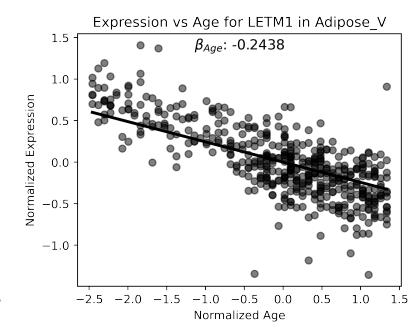
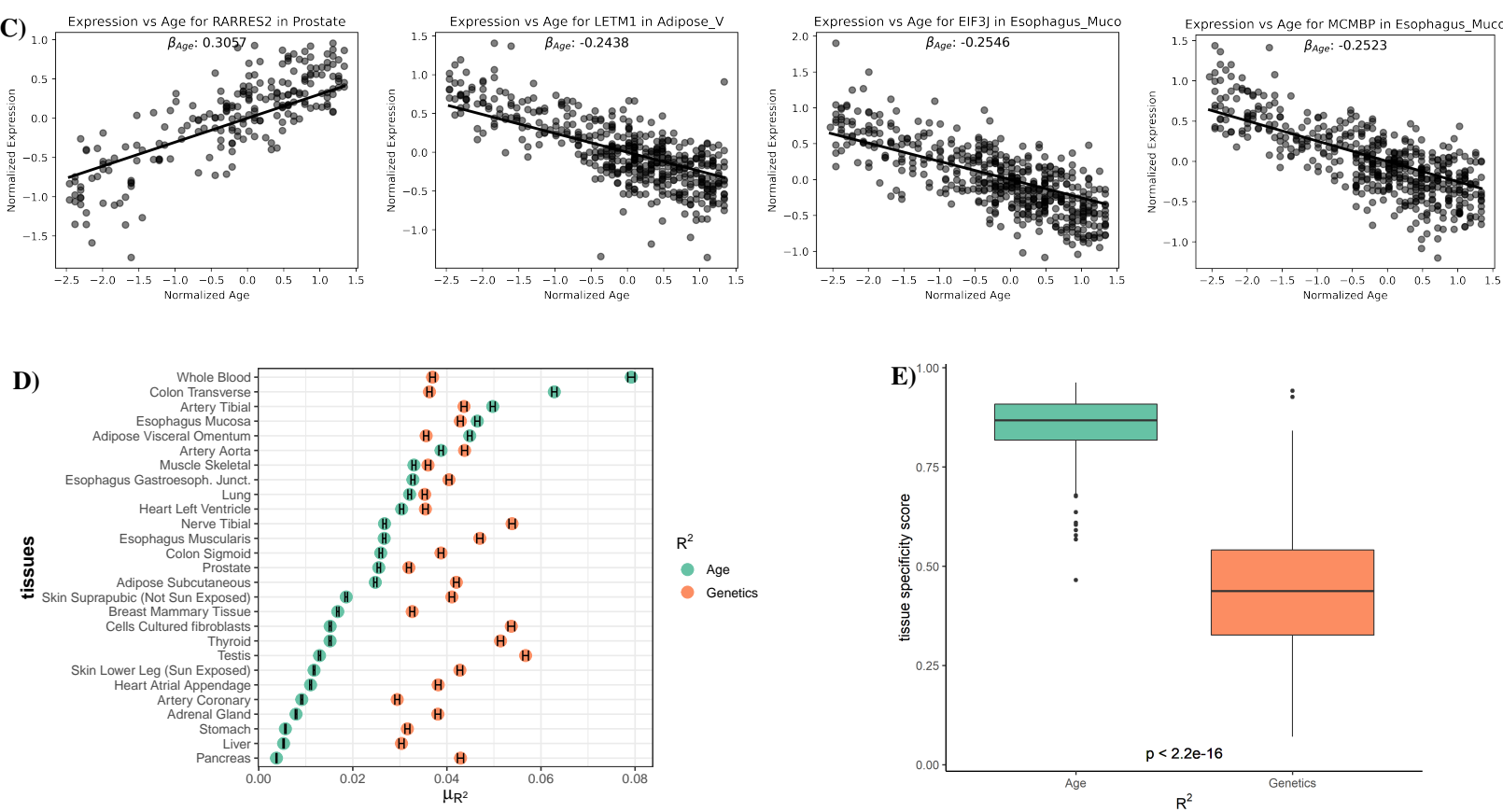

Fig. 3. A joint predictive model of gene expression identifies tissue-specific contributions of age and genetics to transcript levels. A) A schematic of our multi-SNP gene expression association model incorporating sample age. Common SNPs around each gene $g$ are used in combination with an individual's age to predict expression within tissue $t$. Using this trained model, variation in gene expression can be separated into three parts: the components explained by genetics $\left(R_{\text {genetics }}^{2}\right.$ or $\left.h^{2}\right)$, by age $\left(R_{\text {age }}^{2}\right)$ and by all other factors $\left(R_{\text {environment }}^{2}\right)$. B) Ternary plot of the proportion of each genes expression variance explained by age , genetics and the environment. C) Plot of normalized expression vs age for four genes with significant age-correlated expression. Line shows fitted $\beta_{\text {age }}$ from regularized linear model. D) Point estimates of the mean $R_{\text {age }}^{2}$ and $h^{2}$ for each tissue, error bar indicating confidence interval for the estimate. E) The tissue specificity score of $R^{2}$ across 27 tissues for each gene from either age genetics.

(18). As expected, across the vast majority of tissues $\beta_{\text {age } 312}$ was significantly negatively correlated with pLI (Fig. 5B, 313 5C), in line with Medawar's hypothesis. However, five tis- 314 sues exhibited significant signatures in the opposite direc- 315 tion including prostate, transverse colon, breast, whole blood, ${ }_{316}$ and lung tissue $\left(\mathrm{P}<10^{-3}\right)$. These five tissues with non-317 Medawarian trends are driven by highly constrained, func- 318 tionally important genes being expressed at a higher rate in ${ }_{319}$ older individuals (Fig. S14). Using $d N / d S$ (19) as an alternative metric of gene constraint yielded highly correlated results $(\mathrm{R}=-0.72, \mathrm{P}=2.5 \mathrm{e}-5$ Fig. $\mathrm{S} 15, \mathrm{~S} 16)$.

To explore why these five tissues might exhibit distinctive ${ }_{223}$ evolutionary signatures of aging we compared the distribu- 324 tion of significant $\beta_{\text {age }}$ parameters between Medawarian and ${ }_{325}$ non-Medawarian tissues among different hallmark pathways 326 (20). We found 11 signatures exhibiting significantly in- 327 creased $\beta_{\text {age }}($ FDR $<0.01)$ compared to non-Medawarian tis- 328 sues (Fig. 5D, 5E) including DNA-damage, TGF- $\beta$ signalling, MYC targets, and epithelial-to-mesenchymal transition pathways most prominently. All of these signatures are broadly correlated with cellular proliferation, differentiation, and cancer. These results highlight that gene expression patterns in tissues and cell-types that proliferate throughout the course of an individuals life may be subjected to distinct evolutionary pressures.

We also explored the relationship between gene expression heritability and constraint. Across all tissues $h^{2}$ was significantly negatively correlated with pLI (27/27 tissues, P-value $<10^{-3}$ ) (Fig. 5F, S17). While this trend was consistent across tissues, intriguingly it was strongest in heart tissues. The exception was liver, which also had the highest average $R_{\text {environment }}^{2}$ among all tissues, which was only nominally significant after multiple test correction $(\mathrm{P}<0.00185)$. These result indicate that genes in which the variance in expression 
A)

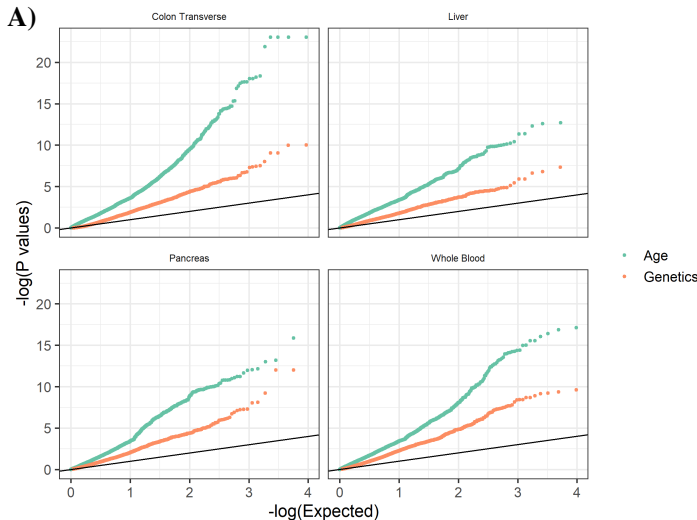

B)

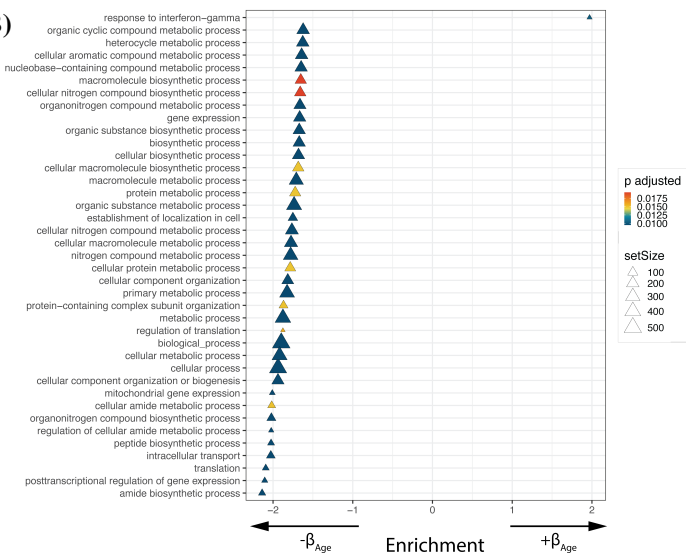

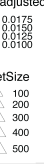

C)

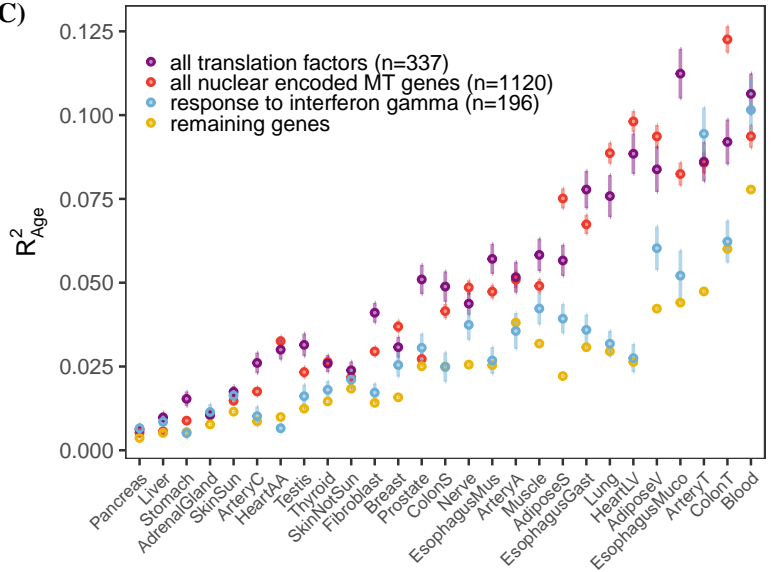

D)

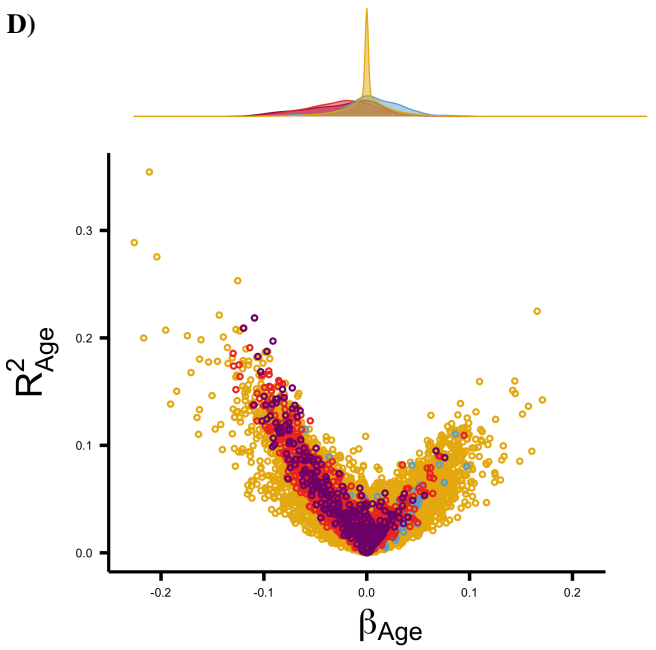

Fig. 4. Functional analysis of age-related genes reveals enriched biological processes. A) $A Q Q$ plot of $p$-values for pathways tested for enrichment using gene set enrichment analysis (GSEA) with genes ranked by either $h^{2}$ or $R_{\text {age }}^{2}$ in four example tissues. B) GSEA enrichments from genes ranked by the mean $\beta_{\text {age }}$ across tissues. Pathways with a correct $P<0.02$ are shown. C) Average gene expression variance explained by age for mitochondrial (MT) genes (red), translation factor genes (purple), interferon gamma genes (blue) and remaining genes (yellow) across all tissues. D) Volcano plot of the variance explained by age vs $\beta_{\text {age }}$ for mitochondrial, translation factors, interferon gamma factors, and remaining genes. Density plot of each axis show on top and right.

is heritable tend to be under significantly less functional con- 349 straint. In contrast, highly conserved genes that are intolerant 350 to mutation are significantly less likely to exhibit heritable 351 variation in gene expression, likely because their expression 352 levels are additionally under constraint.

\section{Discussion}

Studying age-associated changes in gene expression provides ${ }^{357}$ critical insights into the underlying biological processes of 358 aging. Here, we set out to quantify the relative contributions ${ }_{359}$ of aging and genetics to gene expression phenotypes across 360 different human tissues. Our study finds that the predictive ${ }_{361}$ power of eQTLs is significantly impacted by age across sev- 362 eral different tissues and that his effect is more pronounced in 363 older individuals. These results extend upon previous work 364 examining blood tissue (4) and highlight the varied impact of ${ }_{365}$ aging on eQTLs among different tissues. We show that this ${ }_{366}$ result is likely to be in part due to an increase in the inter- ${ }_{367}$ individual heterogeneity of gene expression patterns among ${ }_{368}$ older individuals, potentially as a result of the increased im- 369 pact of the environment. However, our study is limited in 370 it's focus on bulk-tissue transcriptomic data. Early evidence from single cell studies already suggests that differences in gene expression heterogeneity vary among cell types of tissues as a function of age $(6,7,21,22)$. While these studies lack sufficient individual sample sizes and genetic diversity for the statistical approaches used herein, it is possible that in the future the availability of larger datasets will facilitate studying these phenomena at the single-cell level. The extensive tissue heterogeneity we observe suggests that patterns of aging will exhibit substantial cell-type specificity.

We also present a novel approach to jointly model the impact of genetics and aging on gene expression variance to parse out the individual contributions of each of these factors. The increased complexity of our model has little impact on its accuracy with our expression heritability estimates strongly correlated with previous heritability measures across all tissues (mean Pearson's r 0.89, Fig. S7). Using this model we show that age exhibits exceptionally varied affects on different tissues, and indeed, in several tissues age contributes more to gene expression variance on average than genetics. These results also highlight a widespread coordinated signature of age-associated decline in mitochondrial and trans- 
A)

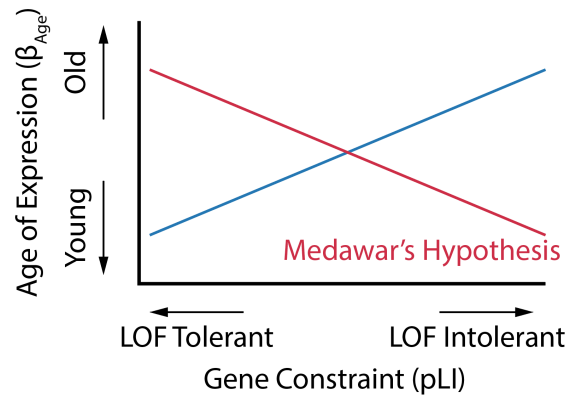

B)

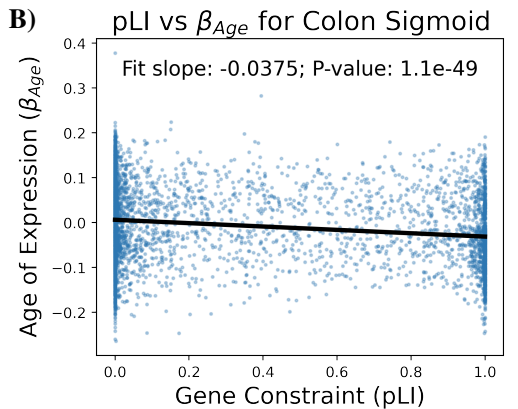

pLI vs $\beta_{\text {Age }}$ for Lung

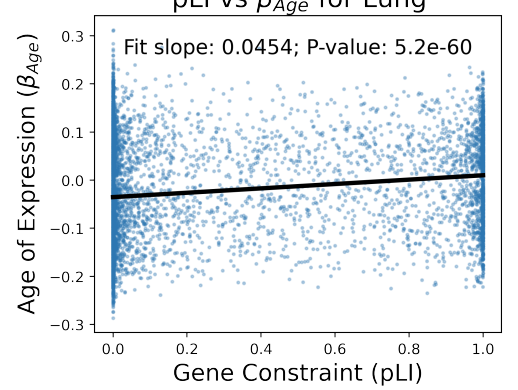

C)

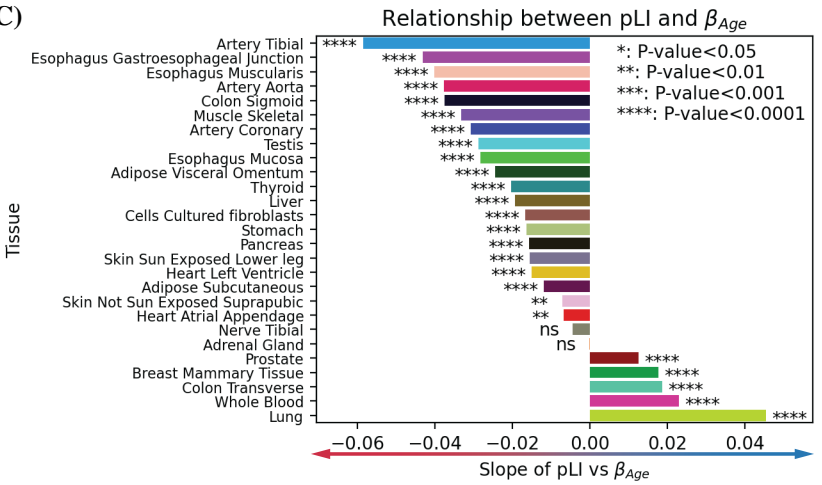

D)

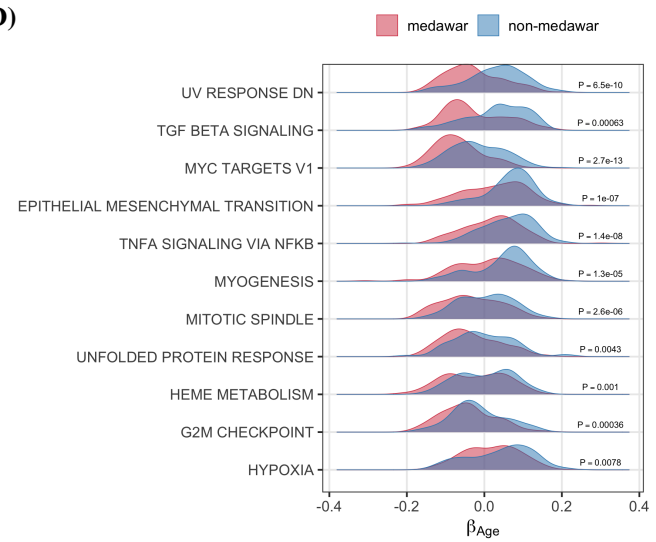

E)

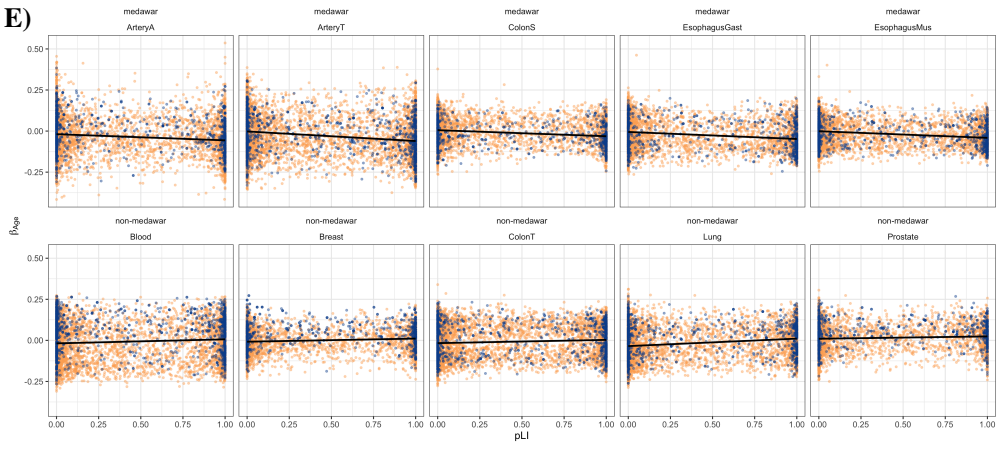

F)

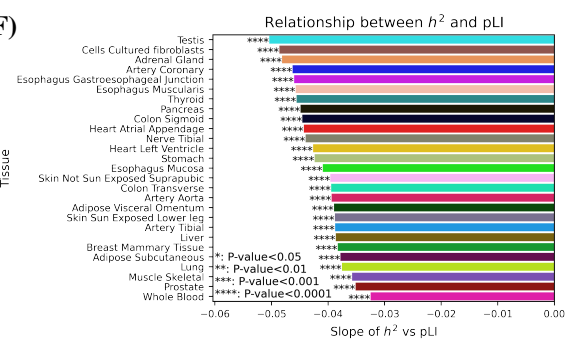

Fig. 5. Tissue-specific evolutionary signatures of aging. A) The expected relationship across genes between the per-gene age-associated slope of gene expression $\left(\beta_{\text {age }}\right)$ and a genes level of constraint (measured by probability loss of function intolerance - pLI) . Medawar's hypothesis predicts a negative relationship (shown in red) between the time of expression and the level of constraint. B) $\beta_{\text {age }}$ across genes plotted as a function of pLI for a tissue exhibiting a Medawarian signature, and a nonMedawarian signature. C) The slope of the relationship between $\beta_{\text {age }}$ and constraint across all tissues. D) Hallmark pathways in which the $\beta_{\text {age }}$ was significantly different between Medawarian and non-Medawarian tissues. E) The relationship between $\beta_{\text {age }}$ and $\mathrm{pLI}$ among tissues showing the strongest Medawarian and non-Medawarian signatures with genes in pathways from (D) highlighted in blue. F) The slope of the relationship between constraint (pLI) and heritability $\left(h^{2}\right)$ across tissues.

lation factors. Dysregulation in mitochondrial function and 385 ribosome biogenesis have been documented as key players 386 in aging, (23, 24), however our results highlight the tissue- 387 specificity of these trends. Our model also allows us to quan- 388 tify the tissue-specific evolutionary context of age-associated gene expression changes. We corroborate the inverse rela- ${ }^{389}$ tionship between age-at-expression and constraint, as pre- ${ }^{390}$ dicted by Medawar's hypothesis and recently documented by ${ }^{391}$ others $(8,9,17)$ across the vast majority of tissues. However, ${ }^{392}$ we also surprisingly identify five tissues which exhibit the op- ${ }^{393}$ posite pattern and show that age-associated signatures of in- ${ }^{394}$ creased proliferation and cancer are enriched in these tissues. ${ }^{395}$ These results highlight the distinct evolutionary forces that ${ }^{396}$ act on late-acting genes expressed in highly proliferative cell- ${ }^{397}$ types. Future work extending these analyses to the single-cell level will provide further insights into the cell-type-specific age-associated patterns of constraint, both in terms of gene expression levels and at the protein-coding level.

Overall this work has several important implications. Our results shed light on recent work on the prediction accuracy of polygenic risk scores (PRS) (25) which found that numerous factors, including age, sex, and socioeconomic status can profoundly impact the prediction accuracy of such scores even in individuals with the same genetic ancestry. Our results highlight that genetics are less predictive of expression phenotypes in several different tissues in older individuals, potentially playing a role in differential PRS accuracy between young and old individuals. This also has important implica- 
tions for disease association and prediction approaches that ${ }_{453}$ leverage expression quantitative trait loci to prioritize vari- 454 ants (e.g. TWAS (26)). If a significant proportion of eQTLs 455 exhibit age-associated biases in their effect size in a tissue of 450 interest, then these approaches may be less powerful when ${ }_{457}$ applied to diseases for which age is a primary risk factor 458 such as heart disease, Alzheimer's dementias, cancers, and ${ }_{459}$ diabetes.

The critical role of aging as a risk factor for many common 460 human diseases underscores the importance of understanding ${ }^{46}$ its impact on cellular systems at the molecular level. Together ${ }^{462}$ our analyses provide novel insights into tissue-specific pat- ${ }^{463}$ terns of aging and the relative impact of genetics and aging 464 on gene expression. We anticipate that future studies across 465 tissues and cells of gene expression, chromatin structure, and 466 epigenetics will further elucidate how both programmed and ${ }^{467}$ stochastic processes of aging drive human disease.

\section{Supplementary Note 1: Methods}

Data collection age groupings. We downloaded gene expression data for multiple individuals and tissues from GTEx V8 (10), which were previously aligned and processed against the hg19 human genome. Tissues were included in ${ }^{472}$ the analysis if they had $>100$ individuals in both the age $\geq 55^{473}$ and $<55$ cohorts described below (Fig. S2). To compare gene expression heritability across individuals of different ages, for some analyses we split the GTEx data for each tissue into two age groups, "young" and "old," based on the median age ${ }_{474}$ of individuals in the full dataset, which was 55 (Fig. S1). ${ }_{475}$ Within each tissue dataset, we then equalized the number of ${ }_{476}$ individuals in the young and old groups by randomly down- ${ }_{477}$ sampling the larger group, to ensure that our models were ${ }_{478}$ equally powered for the two age groups.

PEER factor analysis. We analyzed existing precomputed PEER factors available from GTEx to check for correlations ${ }_{481}$ between these hidden covariates and age. In particular, we ${ }_{482}$ fit a linear regression between age and each hidden covariate ${ }_{483}$ and identified significant age correlations using an F-statistic ${ }_{484}$ (Fig. S3). Because some of the covariates were correlated 485 with age, we generated new age-independent hidden covari- 486 ates of gene expression to remove batch and other confound- ${ }_{487}$ ing effects on gene expression while retaining age related ${ }_{488}$ variation. In particular, we first removed age contributions ${ }_{489}$ to gene expression by regressing gene expression on age and then ran PEER on the age-independent residual gene expres- 490 sion to generate 15 age-independent hidden PEER factors.

Quantifying the effect of eQTLs on gene expression in 493 different age groups. Using the binary age groups defined 494 above, we assessed the relative significance of eQTLs in old 495 and young individuals by carrying out separate assessment of 496 eQTLs identified by GTEx. For each gene in each tissue and each age group, we regressed the GTEx pre-normalized expression levels on the genotype of the lead SNP (identified by 497 GTEx) using 5 PCs, 15 PEER factors, sex, PCR protocol and 498 sequencing platform as covariates, following the GTEx best 499 practices. We confirmed our results using both our recomputed PEER factors as well as the PEER factors provided by GTEx (Fig. S 4). To test for significant differences in genetic associations with gene expression between the old and young age groups, we compared the p-value distributions between these groups for all genes and all SNPs in a given tissue using Welch's t-test.

Jensen-Shannon Divergence as a distance metric between transcriptome profiles. To quantify differences in gene expression between individuals, we computed the pairwise distance for all pairs of individuals in an age group using the square root of Jensen-Shannon Divergence (JSD) distance metric, which measures the similarity of two probability distributions. Here we applied JSD between pairs of individuals' transcriptome vectors containing the gene expression values for each gene, which we converted to a distribution by normalizing by the sum of the entries in the vector. For two individuals' transcriptome distributions, the JSD can be calculated as:

$$
\operatorname{JSD}\left(P_{1}, P_{2}\right)=H\left(\frac{1}{2} P_{1}+\frac{1}{2} P_{2}\right)-\frac{1}{2}\left(H\left(P_{1}\right)+H\left(P_{2}\right)\right)
$$

where $P_{i}$ is the distribution for individual $i$ and $\mathrm{H}$ is the Shannon entropy function:

$$
H(X)=-\sum_{i=1}^{n} P\left(x_{i}\right) \log _{2}\left(P\left(x_{i}\right)\right)
$$

JSD is known to be a robust metric that is less sensitive to noise when calculating distance compared to traditional metrics such as Euclidean distance and correlation. It has been shown that JSD metrics and other approaches yield similar results but that JSD is more robust to outliers (12). The square root of the raw JSD value follows the triangle inequality, enabling us to treat it as a distance metric.

Slope of JSD versus age. In addition to comparing JSD between the two age groups defined above, "young" and "old", we also binned all GTEx individuals into 6 age groups, from 20 to 80 years old with an increment of 10 years. We then computed pairwise distance and average age for each pair of individuals within each bin using the square root of JSD as the distance metric. We applied a linear regression model of JSD versus age to obtain slopes, confidence intervals, and p-values.

Multi-SNP gene expression prediction. We used a multiSNP gene expression prediction model based on PrediXcan (14) to corroborate our findings from the eQTL and JSD analyses on the two age groups, "young" and "old". For each gene in each tissue, we trained a multi-SNP model separately within each age group to predict individual-level gene expression.

$$
Y_{g, t}=\sum_{i} \beta_{i, g, t} X_{i}+\epsilon
$$

Where $\beta_{i, g, t}$ is the coefficient or effect size for SNP $X_{i}$ in gene $g$ and tissue $t$ and $\epsilon$ includes all other noise and environmental effects. The regularized linear model for each gene 
considers dosages of all common SNPs within 1 megabase ${ }_{547}$ of the gene's TSS as input, where common SNPs are de- 548 fined as MAF $>0.05$ and Hardy-Weinberg equilibrium $\mathrm{P}>$ 0.05 . We removed covariate effects on gene expression prior to model training by regressing out both GTEx covariates and age-independent PEER factors (described above). Coefficients were fit using an elastic net model which solves the problem ((27)):

$\min _{\beta_{0}, \beta} \frac{1}{2 N} \sum_{j=1}^{N}\left(Y_{j}-\beta_{0}-X_{j}^{T} \beta\right)^{2}+\lambda\left(\frac{1-\alpha}{2}\|\beta\|_{2}^{2}+\alpha\|\beta\|_{1}{ }_{553}^{552}\right.$

(4) 554

The minimization problem contains both the error of our 555 model predictions $\left(Y_{j}-\beta_{0}-X_{j}^{T} \beta\right)^{2}$ and a regularization 556 term $\lambda\left(\frac{1-\alpha}{2}\|\beta\|_{2}^{2}+\alpha\|\beta\|_{1}\right)$ to prevent model overfitting. ${ }^{557}$ The elastic net regularization term incorporates both L1 ${ }^{558}$ $\left(\|\beta\|_{1}\right)$ ) and L2 $\left(\|\beta\|_{2}^{2}\right)$ penalties. Following PrediXcan,

we weighted the L1 and L2 penalties equally using $\alpha=0.5559$ (14). For each model, the regularization parameter $\lambda$ was 560 chosen via 10 -fold cross validation. The elastic net models ${ }^{561}$ were fit using Python's glmnet package and $R^{2}$ was eval- ${ }^{562}$ uated using scikit-learn. From the trained models for each ${ }^{563}$ gene, we evaluated training set genetic $R^{2}$ (or $h^{2}$ ) for the two ${ }^{564}$ age groups and subtracted $h_{\text {young }}^{2}-h_{\text {old }}^{2}$ to get the differ- 565 ence in gene expression heritability between the groups. We ${ }^{566}$ compared this average difference in heritability to the mean ${ }^{567}$ $\mathrm{JSD}_{\text {old }}-\mathrm{JSD}_{\text {young }}$ and $\log \left(P_{\text {old }}\right)-\log \left(P_{\text {young }}\right)$ using P- 568 values from the eQTL analyses across genes.

Joint model for expression prediction using SNPs and ${ }^{57}$ age. To uncover linear relationships between gene expres- ${ }^{572}$ sion and both age and genetics, we built a set of gene expres- ${ }^{573}$ sion prediction models using both common SNPs and stan- ${ }^{574}$ dardized age as input. An individual's gene expression level $Y$ for a gene $g$ and tissue $t$ is modeled as:

$$
Y_{g, t}=\sum_{i} \beta_{i, g, t} X_{i}+\beta_{\text {age }, g, t} A+\epsilon
$$

Where $A$ is the normalized age of an individual. Coefficients 580 were fit using elastic net regularization, as above, which sets 581 coefficients for non-informative predictors to zero. The sign of the fitted age coefficient $\left(\beta_{\text {age,g,t }}\right)$, when nonzero, reflects ${ }^{582}$ whether the gene in that tissue is expressed more in young ${ }^{583}$ (negative coefficient) or old (positive coefficient) individuals. ${ }^{584}$ We also evaluated the training set $R^{2}$ using the fit model separately for genetics (across all SNPs in the model) and age. 586 To check consistency of tissue-specific gene expression her- ${ }_{588}^{587}$ itability estimates from our model and the original PrediXcan model trained on GTEx data, we evaluate Pearson's $r$ be- ${ }_{590} 589$ tween our heritability estimates and those of PrediXcan (Fig. ${ }^{591}$ S7), using heritability estimates from the original PrediXcan ${ }^{592}$ model available in PredictDB.

Tissue specificity of age and genetic associations. 594 We evaluated the variability of age and genetic associations ${ }_{596}^{595}$ across tissues using a measure of tissue specificity for age ${ }_{597}^{596}$ and genetic $R^{2}$ (28). We measured the tissue-specificity of a gene $g$ 's variance explained $R_{g}^{2}$ using the following metric:

$$
S_{g}=\frac{\sum_{t=1}^{n}\left(1-\frac{R_{g, t}^{2}}{R_{g, \max }^{2}}\right)}{n-1}
$$

Where $n$ is the total number of tissues, $R_{g, t}^{2}$ is the variance explained by either age or genetics for the gene $g$ in tissue $t$ and $R_{g, \max }^{2}$ is the maximum variance explained for $g$ over all tissues. This metric can be thought of as the average reduction in variance explained relative to the maximum variance explained across tissues for a given gene. The metric ranges from 0 to 1 , with 0 representing ubiquitously high genetic or age $R^{2}$ and 1 representing only one tissue with nonzero genetic or age $R^{2}$ for a given gene. We calculate $S_{g}$ separately for $R_{\text {age }}^{2}$ and $R_{\text {genetics }}^{2}$ across all genes.

Functional constraint analysis. We quantified gene constraint using probability of loss of function intolerance (pLI) from gnomAD 2.1.1 (18). We analyzed the relationships between pLI vs $\beta_{\text {age }}$ and pLI vs heritability across genes. For these analyses, genes were only included if age or genetics were predictive of gene expression $\left(R^{2}>0\right)$ for that gene. For genes with $R^{2}>0$, we used linear regression to determine the direction of the relationship between pLI and $\beta_{\text {age }}$ and heritability for each tissue. The F-statistic was used to determine whether pLI was significantly related to these two model outputs. For pLI vs $\beta_{\text {age }}$, a significant negative slope was considered a Medawar trend (consistent with the Medawar hypothesis) and a significant positive slope a non-Medawar trend. We also analyzed the evolutionary constraint metric $\mathrm{dN} / \mathrm{dS}$ (19) and its tissue-specific relationship with $\beta_{\text {age }}$ by determining the slope and significance of the linear regression, as above.

Non-Medawar tissue analysis. To explore the nonMedawar trend in some tissues, we assessed the distribution of $\beta_{\text {age }}$ across Medawar and non-Medawar tissues for genes within each of the 50 MSigDB hallmark pathways (20). Significant differences between the distributions were called using a t-test, and p-values were adjusted for multiple hypothesis testing using a Benjamini-Hochberg correction.

Code availability. All analyses were performed in $\mathrm{R}$ version 4.0.2 and Python 3.6. All code is available online at https://github.com/sudmantlab/gene_expression_aging.

\section{ACKNOWLEDGEMENTS}

This work was supported by the National Institute of General Medical Sciences grant R35GM142916 to P.H.S. and the National Human Genome Research Institute grant R00HG009677 to N.M.I.

\section{AUTHOR CONTRIBUTIONS}

$\mathrm{RY}, \mathrm{RC}, \mathrm{JMV}, \mathrm{HS}, \mathrm{PS}$, and PHS performed all analysis. RY, RC, NMI, and PHS wrote the manuscript. PHS and NMI supervised the project. PHS conceived of the project.

\section{Bibliography}

1. B Charlesworth, Fisher, medawar, hamilton and the evolution of aging. Genetics 156, 927931 (2000).

2. MR Rose, CL Rauser, G Benford, M Matos, LD Mueller, HAMILTONS FORCES OF NATURAL SELECTION AFTER FORTY YEARS. Evolution 61, 1265-1276 (2007). 
3. A Viñuela, et al., Age-dependent changes in mean and variance of gene expression across tissues in a twin cohort. Hum. Mol. Genet. 27, 732-741 (2017).

4. B Balliu, et al., Genetic regulation of gene expression and splicing during a 10-year period of human aging. Genome Biol. 20 (2019).

5. M Somel, P Khaitovich, S Bahn, S Pääbo, M Lachmann, Gene expression becomes heterogeneous with age. Curr. Biol. 16, R359-R360 (2006).

6. S Wang, et al., Single-cell transcriptomic atlas of primate ovarian aging. Cell 180, 585600.e19 (2020).

7. CP Martinez-Jimenez, et al., Aging increases cell-to-cell transcriptional variability upon immune stimulation. Science 355, 1433-1436 (2017).

8. C Cheng, M Kirkpatrick, Molecular evolution and the decline of purifying selection with age. Nat. Commun. 12 (2021).

9. K Jia, C Cui, Y Gao, Y Zhou, Q Cui, An analysis of aging-related genes derived from the genotype-tissue expression project (GTEx). Cell Death Discov. 4 (2018).

10. Genetic effects on gene expression across human tissues. Nature 550, 204-213 (2017).

11. O Stegle, L Parts, R Durbin, J Winn, A bayesian framework to account for complex nongenetic factors in gene expression levels greatly increases power in eQTL studies. PLoS Comput. Biol. 6, e1000770 (2010).

12. PH Sudmant, MS Alexis, CB Burge, Meta-analysis of RNA-seq expression data across species, tissues and studies. Genome Biol. 16 (2015).

13. P Sen, PP Shah, R Nativio, SL Berger, Epigenetic mechanisms of longevity and aging. Cell 166, 822-839 (2016).

14. ER Gamazon, et al., A gene-based association method for mapping traits using reference transcriptome data. Nat. Genet. 47, 1091-1098 (2015).

15. A Subramanian, et al., Gene set enrichment analysis: A knowledge-based approach for interpreting genome-wide expression profiles. Proc. Natl. Acad. Sci. 102, 15545-15550 (2005).

16. S Rath, et al., Mitocarta3.0: an updated mitochondrial proteome now with sub-organelle localization and pathway annotations. Nucleic Acids Res. 49, D1541-D1547 (2020).

17. R Cui, et al., Relaxed selection limits lifespan by increasing mutation load. Cell 178, 385-399.e20 (2019).

18. M Lek, et al., Analysis of protein-coding genetic variation in 60,706 humans. Nature 536, 285-291 (2016).

19. M Gayà-Vidal, M Albà, Uncovering adaptive evolution in the human lineage. BMC Genomics 15, 599 (2014).

20. A Liberzon, et al., The molecular signatures database hallmark gene set collection. Cell Syst. 1, 417-425 (2015)

21. N Almanzar, et al., A single-cell transcriptomic atlas characterizes ageing tissues in the mouse. Nature 583, 590-595 (2020).

22. $\mathrm{P}$ Cheung, et al., Single-cell chromatin modification profiling reveals increased epigenetic variations with aging. Cell 173, 1385-1397.e14 (2018).

23. S Srivastava, The mitochondrial basis of aging and age-related disorders. Genes $\mathbf{8}, 398$ (2017).

24. S Tahmasebi, A Khoutorsky, MB Mathews, N Sonenberg, Translation deregulation in human disease. Nat. Rev. Mol. Cell Biol. 19, 791-807 (2018).

25. H Mostafavi, et al., Variable prediction accuracy of polygenic scores within an ancestry group. eLife 9 (2020).

26. M Wainberg, et al., Opportunities and challenges for transcriptome-wide association studies. Nat. Genet. 51, 592-599 (2019).

27. JH Friedman, T Hastie, R Tibshirani, Regularization paths for generalized linear models via coordinate descent. J. Stat. Softw. 33, 122 (2010).

28. I Yanai, et al., Genome-wide midrange transcription profiles reveal expression level relationships in human tissue specification. Bioinformatics 21, 650-659 (2004). 


\section{Supplementary Figures}

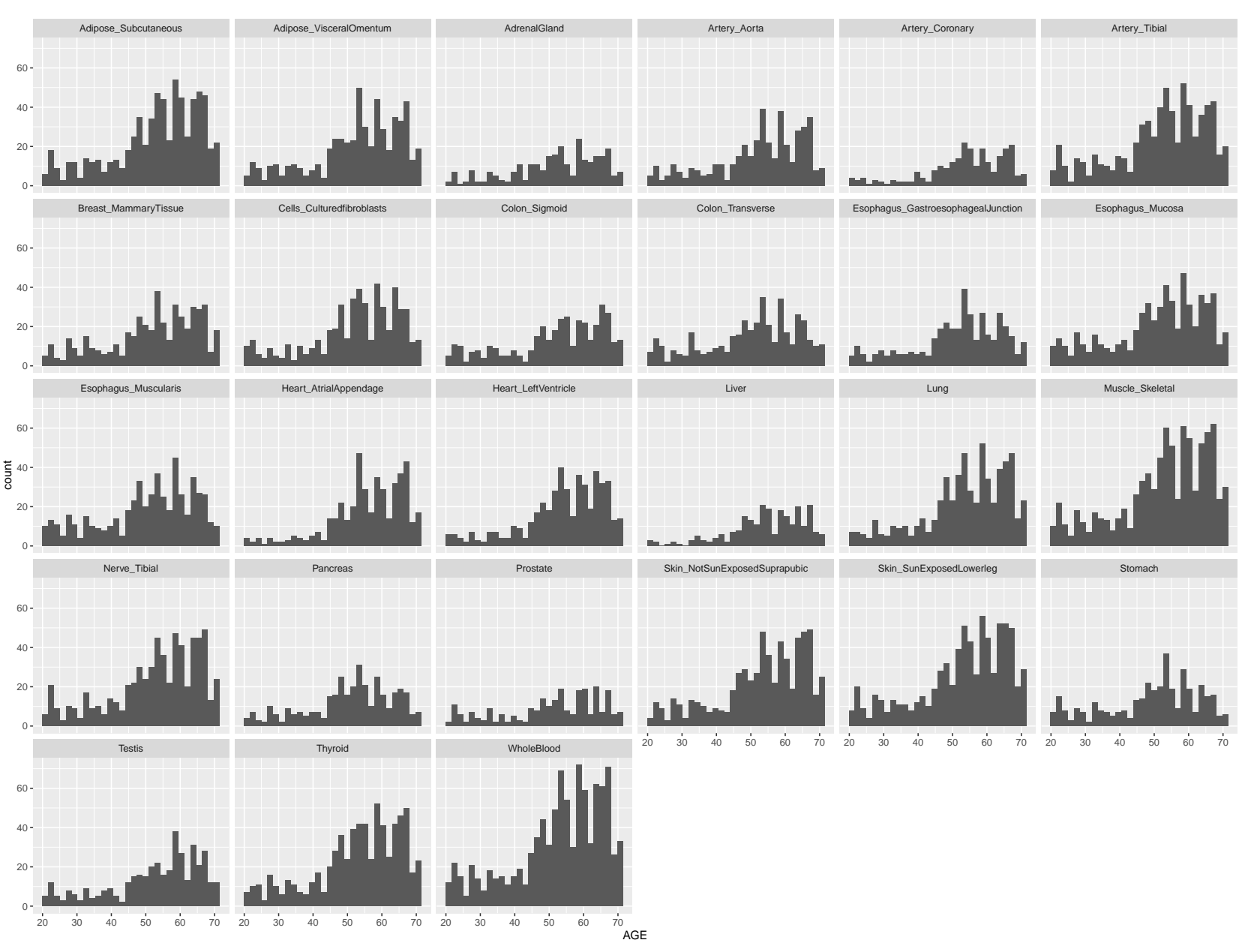

Fig S 1. Sample distribution of each GTEx tissue by age 
bioRxiv preprint doi: https://doi.org/10.1101/2021.11.16.468753; this version posted November 19, 2021. The copyright holder for this preprint (which was not certified by peer review) is the author/funder. All rights reserved. No reuse allowed without permission.

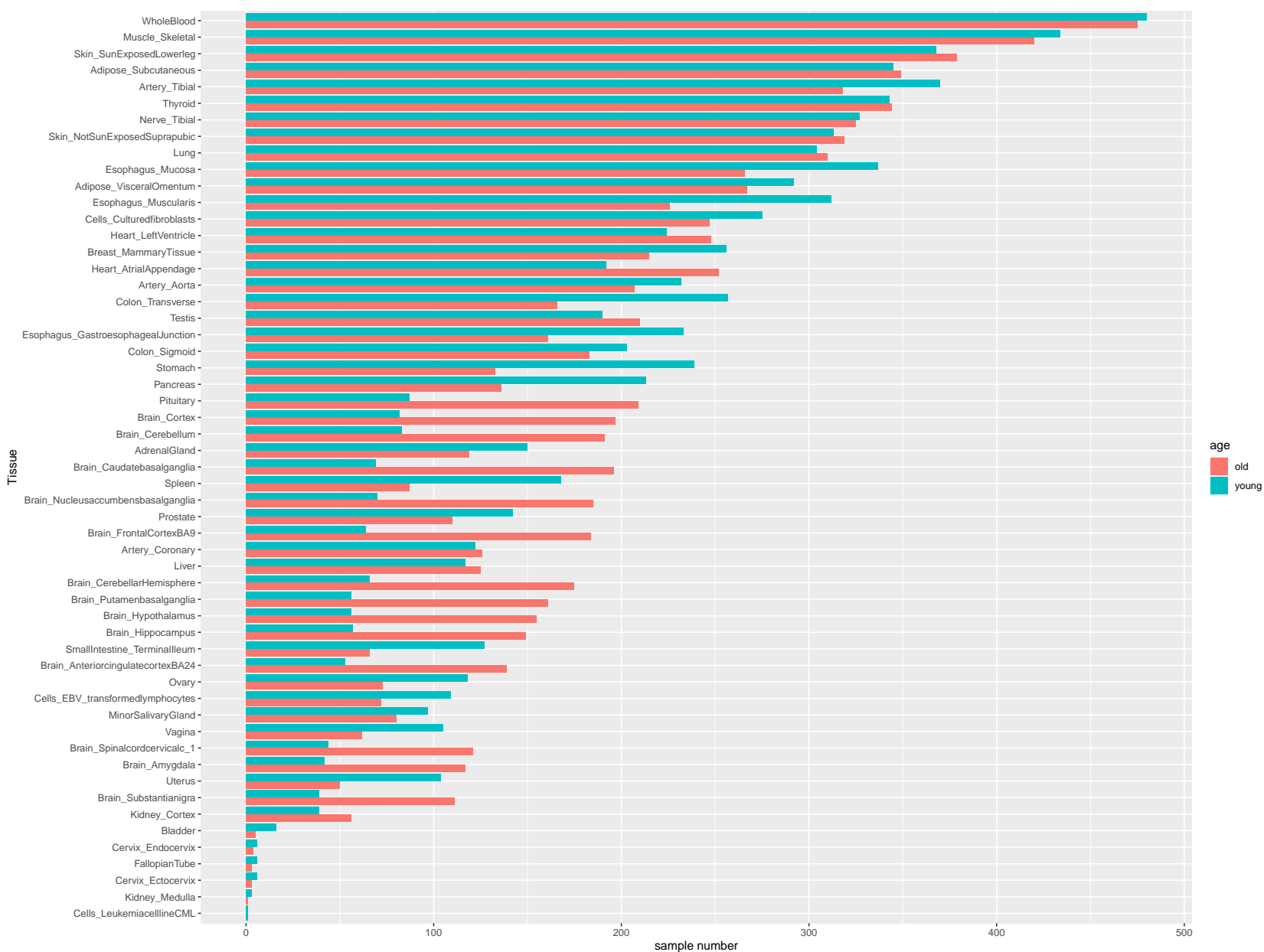

Fig S 2. Sample number for individuals above and below the median age by tissue for 47 GTEx tissues

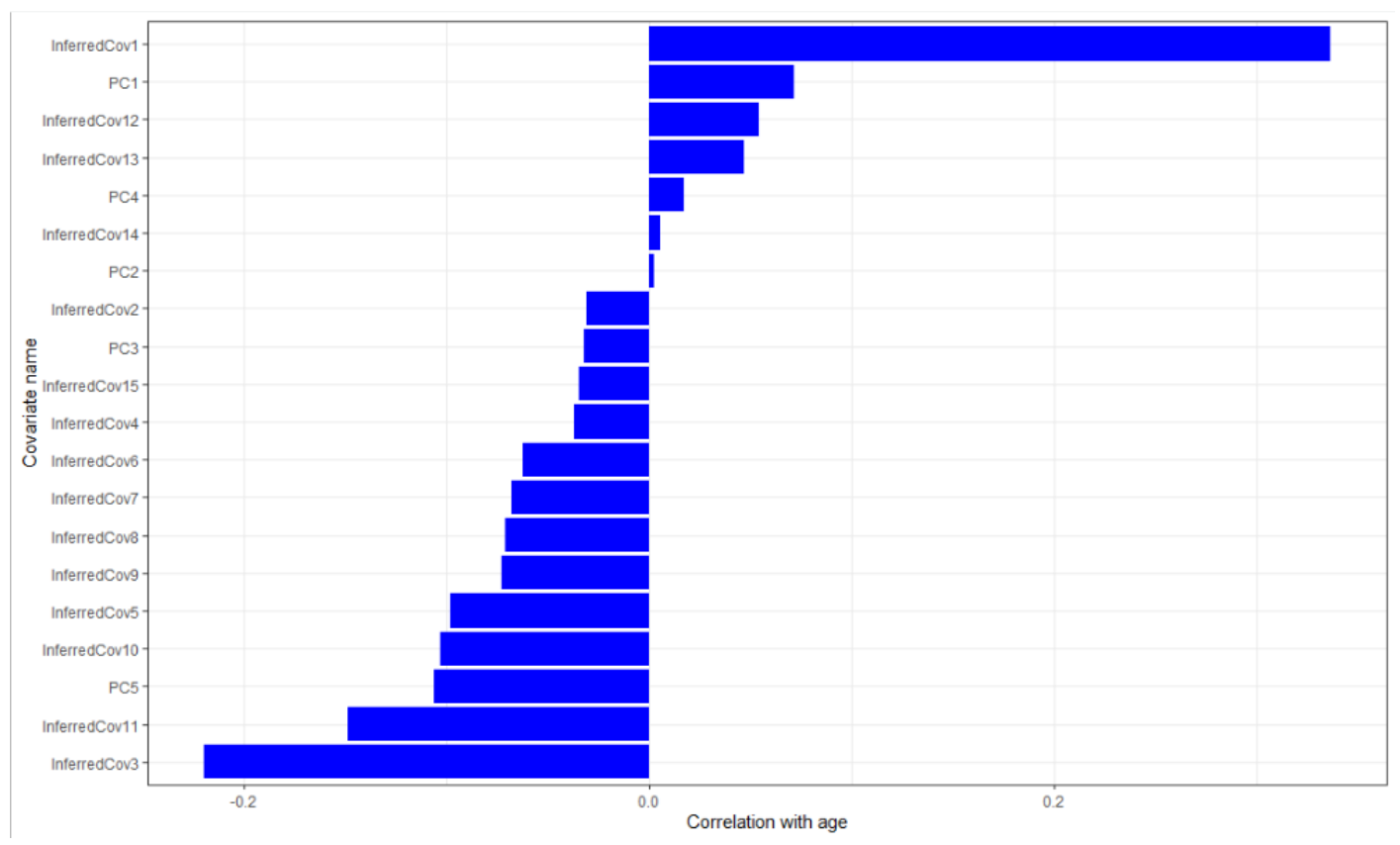

Fig S 3. Correlation of GTEx covariates with age. 

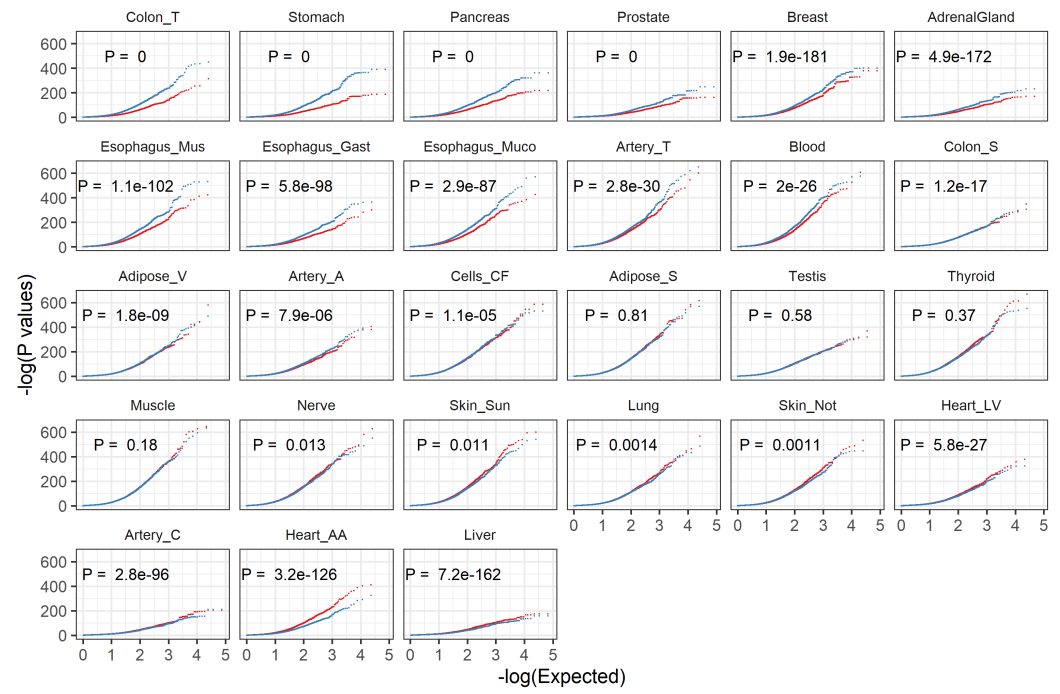

Fig S 4. QQ plot of eQTL p-values for old (red) and young (blue) cohorts across 27 tissues using GTEx PEER factors. Significant differences between $p$-value distributions annotated for each tissue.

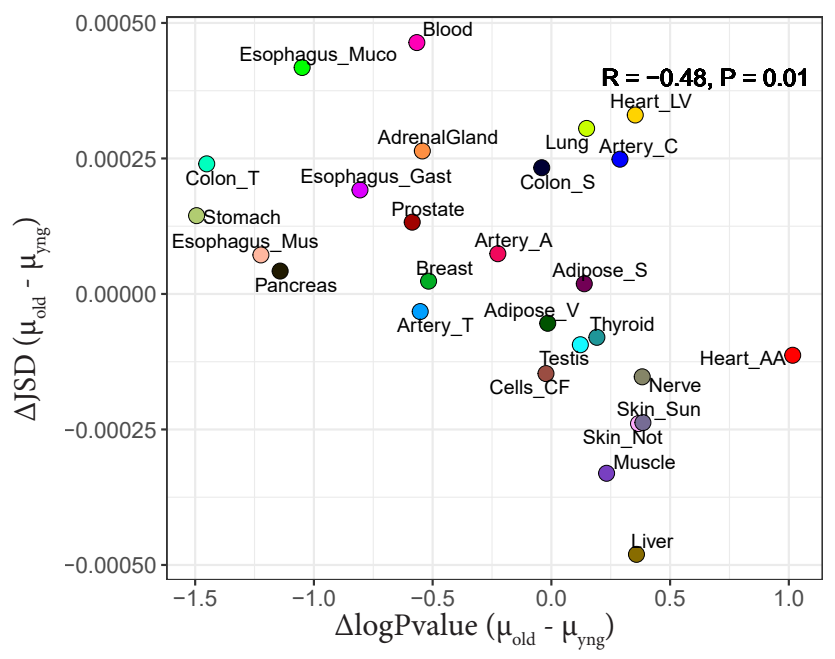

Fig S 5. Scatter plot showing the correlation between the difference in average JSD between young and old individuals and difference in eQTL log(p-value) between young and old

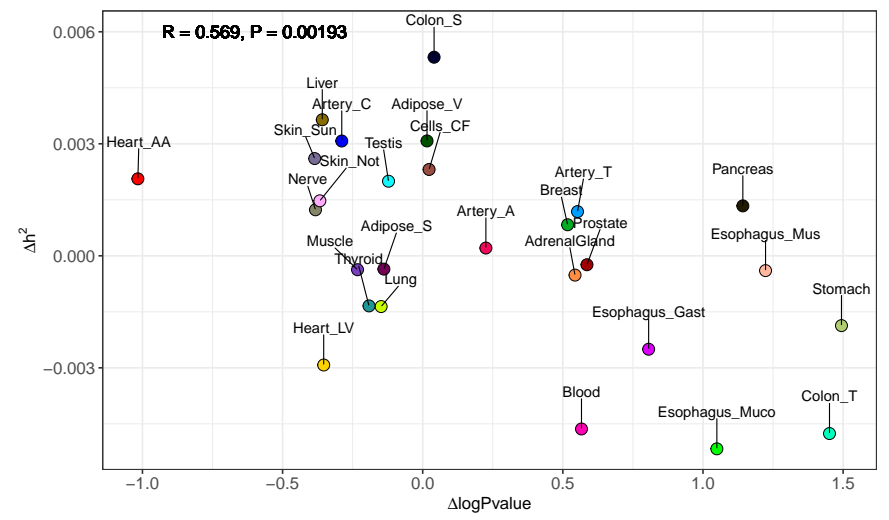

Fig S 6. High correspondence of age-related changes in gene expression associations for single SNP and multi-SNP models. Scatter plot showing the correlation between the difference in heritability estimated by the multi-SNP linear model (PrediXcan) in young and old individuals and difference in eQTL $\log (p$-value) in young and old individuals 
bioRxiv preprint doi: https://doi.org/10.1101/2021.11.16.468753; this version posted November 19, 2021. The copyright holder for this preprint (which was not certified by peer review) is the author/funder. All rights reserved. No reuse allowed without permission.

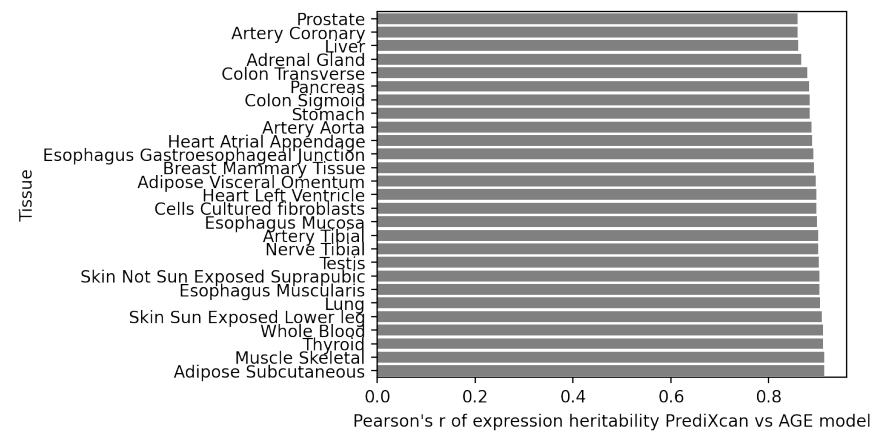

Fig S 7. Pearson's $r$ of heritability estimate from PrediXcan (PredictDB) vs our model for each tissue
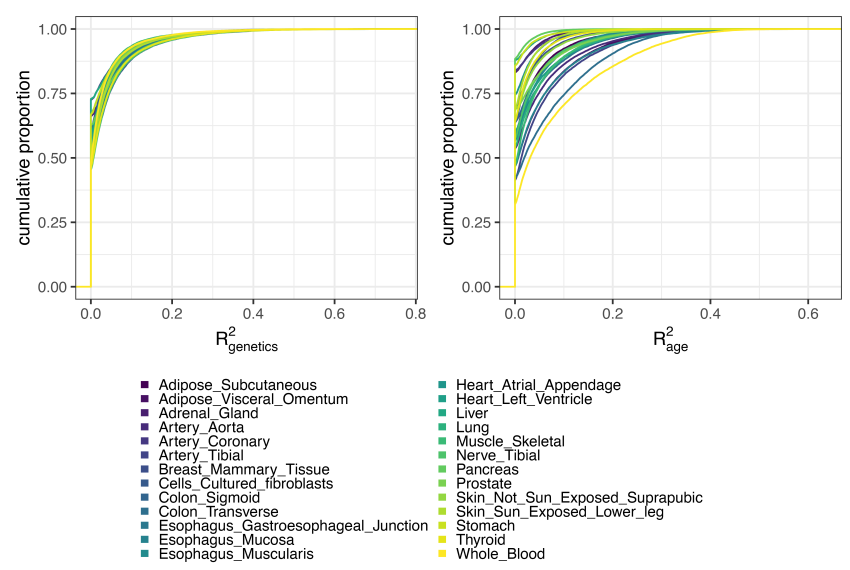

Fig S 8. Cumulative distribution of $R_{\text {age }}^{2}$ and $h^{2}$ for all modeled genes within 27 tissues.

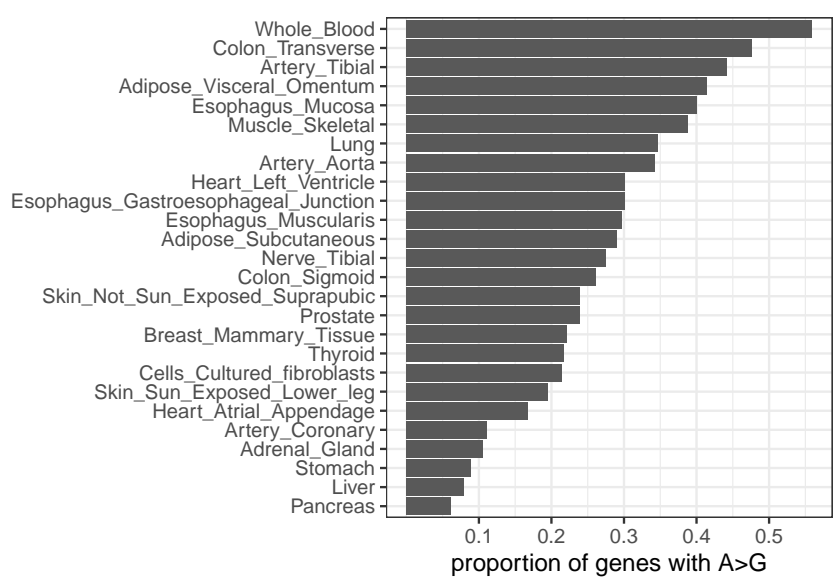

Fig S 9. Proportion of genes within a tissue that have $R_{\text {age }}^{2}>h^{2}$ 
bioRxiv preprint doi: https://doi.org/10.1101/2021.11.16.468753; this version posted November 19, 2021. The copyright holder for this preprint (which was not certified by peer review) is the author/funder. All rights reserved. No reuse allowed without permission.

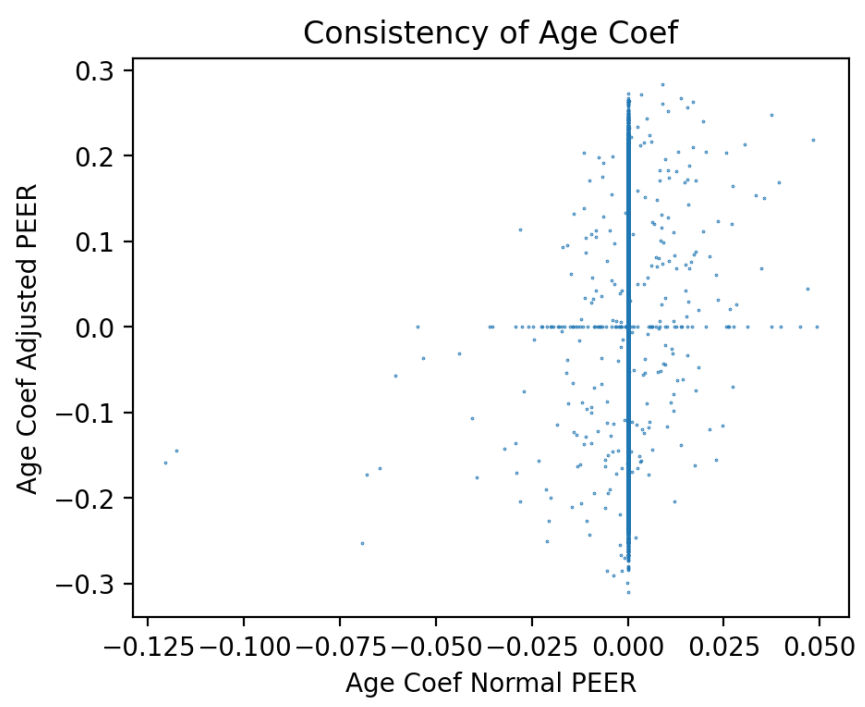

Fig S 10. Scatter plot of each gene's $\beta_{\text {age }}$ of multiSNP model using GTEx PEER factors vs age-independent PEER factors for Whole Blood
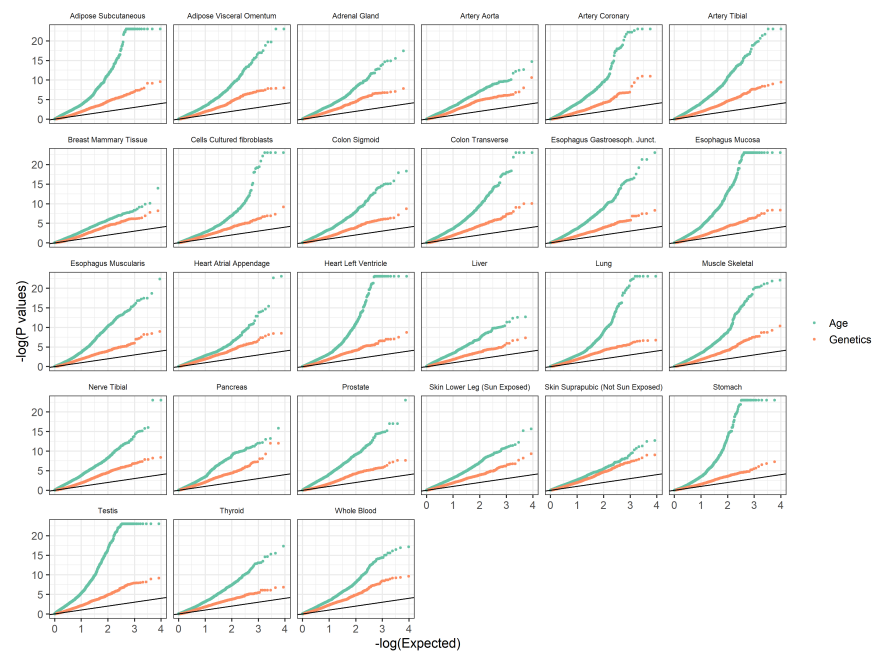

Fig S 11. GO gene set enrichment P-values across all tissue for genes ranked by either $h^{2}$ or $R_{\text {age }}^{2}$ 
bioRxiv preprint doi: https://doi.org/10.1101/2021.11.16.468753; this version posted November 19, 2021. The copyright holder for this preprint (which was not certified by peer review) is the author/funder. All rights reserved. No reuse allowed without permission.

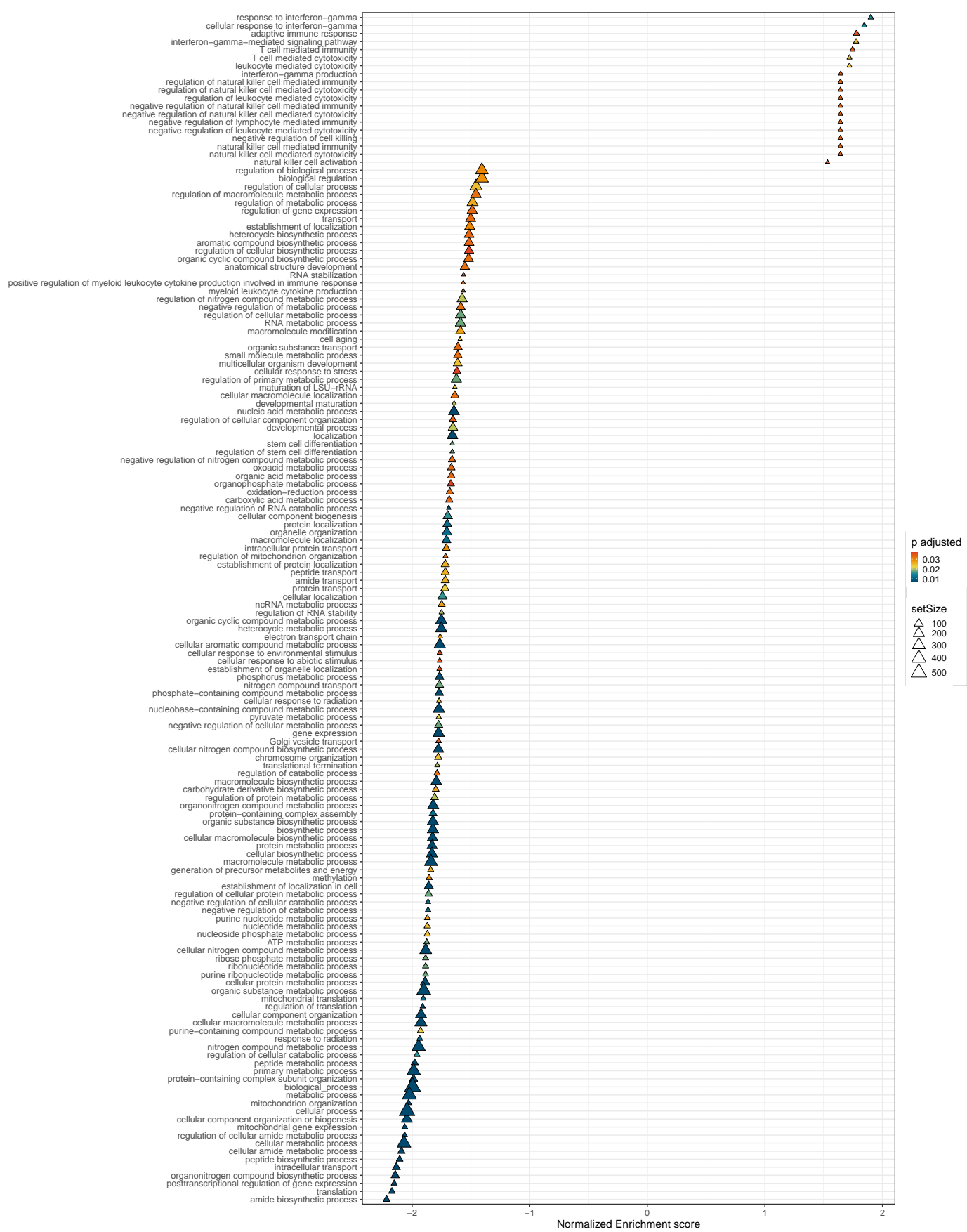

Fig S 12. GO Biological Processes common enrichment in all tissues with genes ranked by average $\beta_{\text {age }}$ (FDR 0.05)

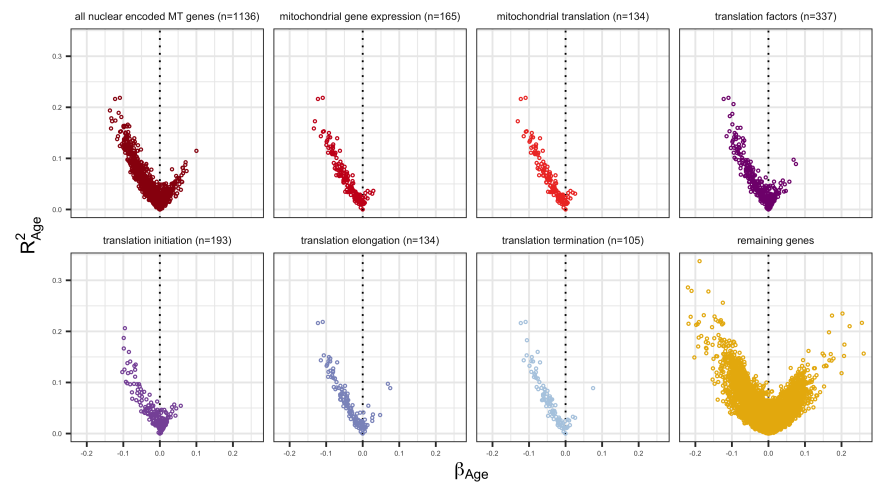

Fig S 13. Relationship between the average $\beta_{\text {age }}$ and average $R^{2}$ across tissues for genes associated with specific mitochondrial and translation pathways 
bioRxiv preprint doi: https://doi.org/10.1101/2021.11.16.468753; this version posted November 19, 2021. The copyright holder for this preprint (which was not certified by peer review) is the author/funder. All rights reserved. No reuse allowed without permission.

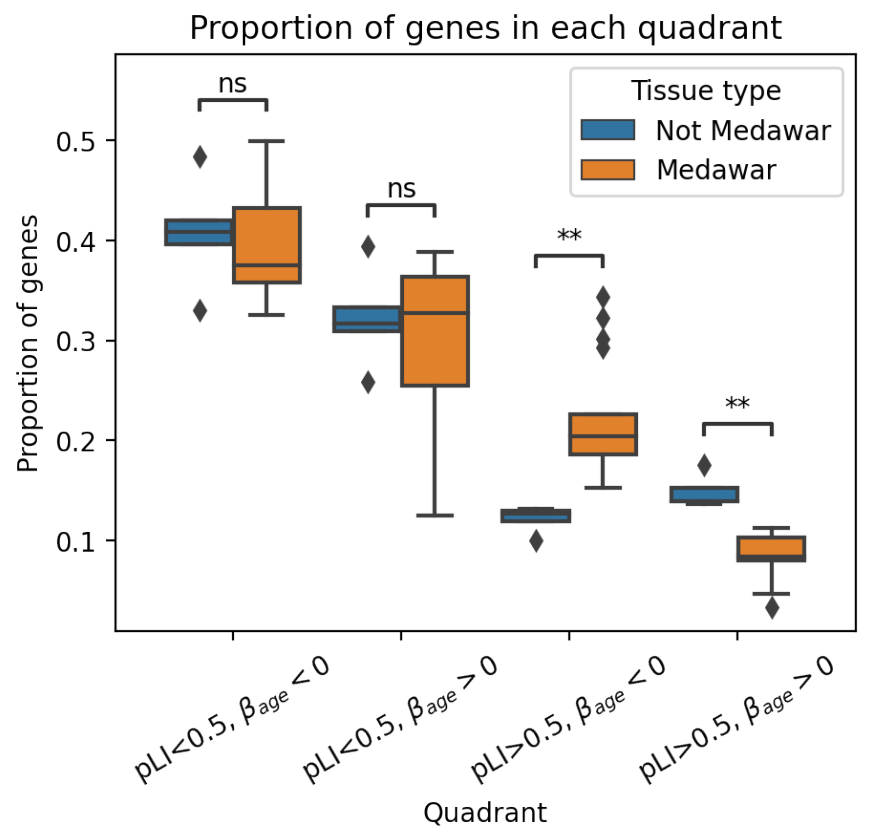

Fig S 14. More highly constrained, late expressed genes in non-Medawar tissues than in Medawar tissues. We plot the proportion of genes within each quadrant of the gene constraint (pLI) vs. age of expression ( $\beta_{\text {age }}$ ) plots stratified by whether the tissue showed a significant Medawar or Non-Medawar trend.

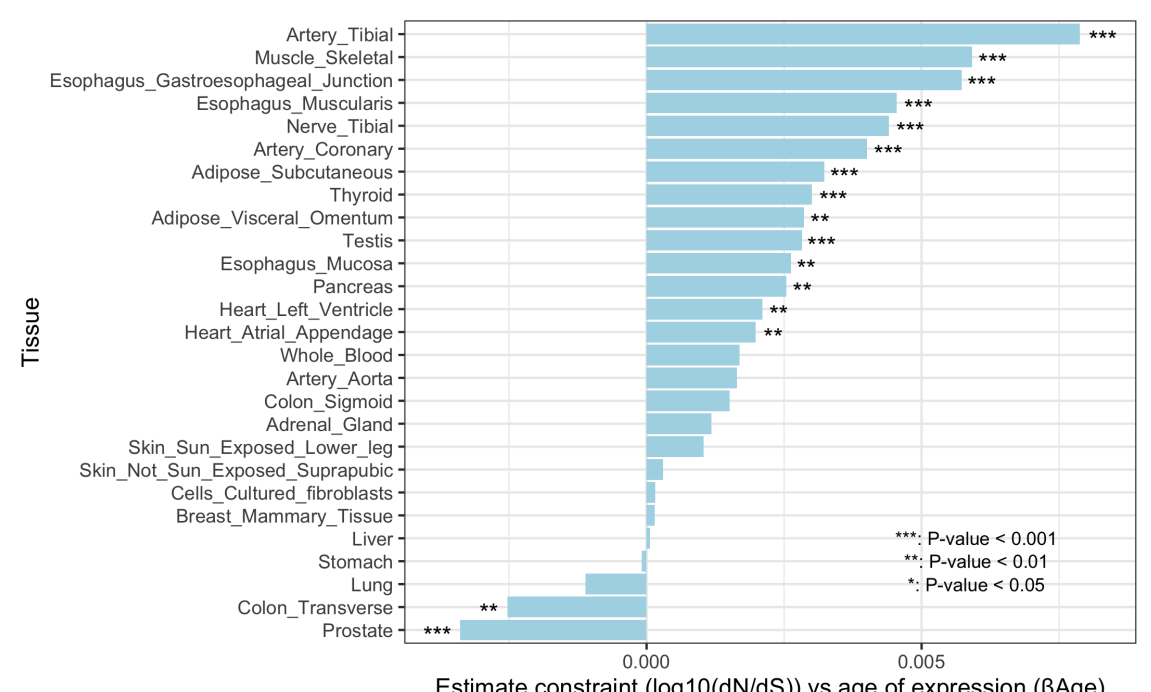

Fig S 15. Linear regression estimates of evolutionary constraint (dN/dS data from ortholog comparison between 8,175 human and chimpanzee genes) vs ageassociated gene expression $\left(\beta_{\text {age }}\right)$ across tissues for $R_{\text {age }}^{2}>0.10 / 27$ tissues have significant $p$-values $(p$-value $<0.001)$ 


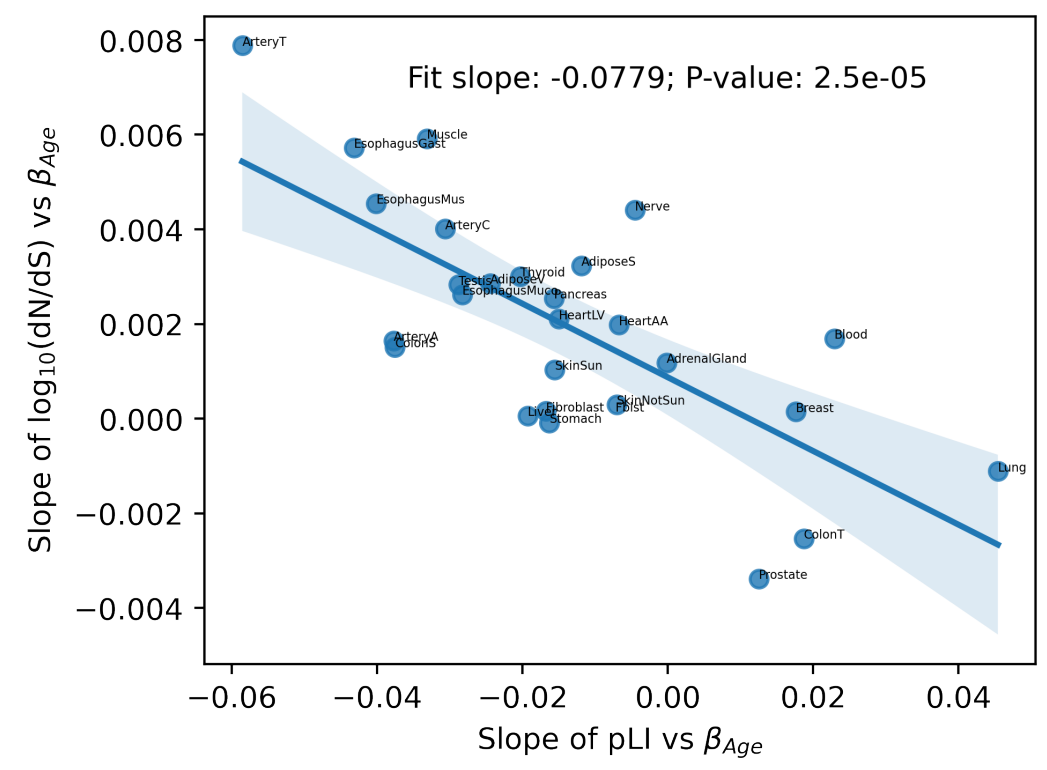

Fig S 16. Consistency of Medawarian trend measures. We plot the slope of gene constraint metrics (pLI and dN/dS) vs $\beta_{\text {age }}$ for each tissue
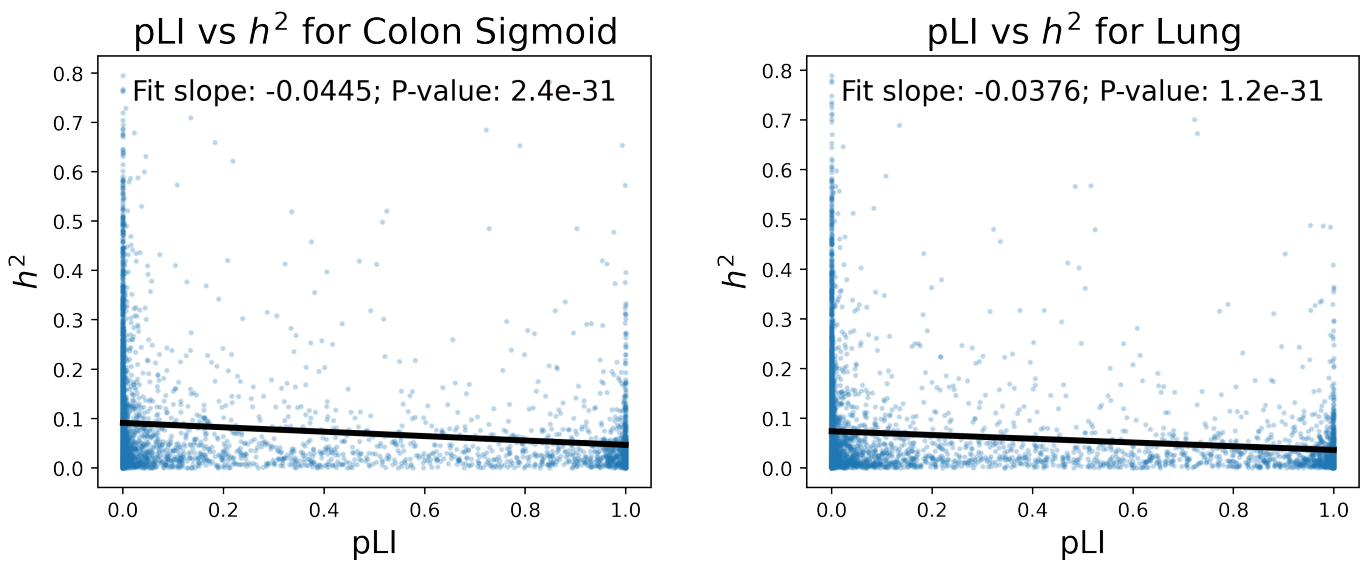

Fig S 17. Gene expression heritability vs $\mathrm{pLI}$ for two tissues 


\begin{tabular}{|l||l|l|}
\hline TISSUE & $\#$ (old individuals) & \#(young individuals) \\
\hline Adipose_Subcutaneous & 349 & 345 \\
Adipose_VisceralOmentum & 267 & 292 \\
AdrenalGland & 119 & 150 \\
Artery_Aorta & 207 & 232 \\
Artery_Coronary & 126 & 122 \\
Artery_Tibial & 318 & 370 \\
Breast_MammaryTissue & 215 & 256 \\
Cells_Culturedfibroblasts & 247 & 275 \\
Colon_Sigmoid & 183 & 203 \\
Colon_Transverse & 166 & 257 \\
Esophagus_GastroesophagealJunction & 161 & 233 \\
Esophagus_Mucosa & 266 & 337 \\
Esophagus_Muscularis & 226 & 312 \\
Heart_AtrialAppendage & 252 & 192 \\
Heart_LeftVentricle & 248 & 224 \\
Liver & 125 & 117 \\
Lung & 310 & 304 \\
Muscle_Skeletal & 420 & 434 \\
Nerve_Tibial & 325 & 327 \\
Pancreas & 136 & 213 \\
Prostate & 110 & 142 \\
Skin_NotSunExposedSuprapubic & 319 & 313 \\
Skin_SunExposedLowerleg & 379 & 368 \\
Stomach & 133 & 239 \\
Testis & 210 & 190 \\
Thyroid & 344 & 343 \\
WholeBlood & 475 & 480 \\
\hline
\end{tabular}

Table S 1. Number of old and young individuals per tissue 


\begin{tabular}{|l||l|}
\hline Tissue & Short name \\
\hline Adipose_Subcutaneous & AdiposeS \\
Adipose_VisceralOmentum & AdiposeV \\
AdrenalGland & AdrenalGland \\
Artery_Aorta & ArteryA \\
Artery_Coronary & ArteryC \\
Artery_Tibial & ArteryT \\
Breast_Mammary_Tissue & Breast \\
Cells_Cultured_fibroblasts & Fibroblast \\
Colon_Sigmoid & ColonS \\
Colon_Transverse & ColonT \\
Esophagus_Gastroesophageal_Junction & EsophagusGast \\
Esophagus_Mucosa & EsophagusMuco \\
Esophagus_Muscularis & EsophagusMus \\
Heart_Atrial_Appendage & HeartAA \\
Heart_Left_Ventricle & HeartLV \\
Liver & Liver \\
Lung & Lung \\
Muscle_Skeletal & Muscle \\
Nerve_Tibial & Nerve \\
Pancreas & Pancreas \\
Prostate & Prostate \\
Skin_Not_Sun_Exposed_Suprapubic & SkinNotSun \\
Skin_Sun_Exposed_Lower_leg & SkinSun \\
Stomach & Stomach \\
Testis & Testis \\
Thyroid & Thyroid \\
Whole_Blood & Blood \\
\hline
\end{tabular}

Table S 2. Tissues with short names 